\title{
Fourier-Based Imaging for Multistatic Radar Systems
}

\author{
Yuri Álvarez, Member, IEEE, Yolanda Rodriguez-Vaqueiro, Student Member, IEEE, \\ Borja Gonzalez-Valdes, Member, IEEE, Spiros Mantzavinos, Carey M. Rappaport, Fellow, IEEE, \\ Fernando Las-Heras, Senior Member, IEEE, and José Ángel Martínez-Lorenzo, Member, IEEE
}

\begin{abstract}
Fourier-based methods for monostatic and bistatic setups have been widely used for high-accuracy radar imaging. However, the multistatic configuration has several characteristics that make Fourier processing more challenging: 1) a nonuniform grid in $k$-space, which requires multidimensional interpolation methods, and 2) image distortion when the incident spherical wave is approximated by a plane wave. This contribution presents a Fourier-based imaging method for multistatic systems, solving the aforementioned limitations: the first, by using $k$-space partitioning and applying interpolation in each domain; the second, by approximating the spherical wave with multiple plane waves. Both solutions are fully parallelizable, thus allowing calculation time savings. Validation and benchmarking with a synthetic aperture radar backpropagation algorithm have been performed through 2-D and 3-D simulation-based examples. Imaging results from radar measurements have been assessed.
\end{abstract}

Index Terms-Fast Fourier transform (FFT), imaging systems, multistatic radar system, synthetic aperture radar (SAR).

\section{INTRODUCTION}

I MAGING techniques based on electromagnetic waves are used in a wide range of systems where nondestructive testing is required. Among these applications, medical diagnostics [1], detection of buried targets [2], or security scanners for detecting concealed weapons [3], [4] are of special interest.

Among many inverse methods for recovering scatterer geometry from the knowledge of the scattered field, inverse synthetic aperture radar (SAR) imaging [5], backward field backpropagation [6], [7], and matched-filtering [8], [9] have been proved to be effective algorithms for high-resolution image formation.

Interest in submillimeter-wave and terahertz frequency bands for imaging applications [10]-[12] requires dealing with electrically large problems of hundreds of wavelengths. For the afore-

Manuscript received November 23, 2013; revised March 08, 2014; accepted June 12, 2014. This work is supported in part by ALERT, an NSF ERC Program, under Award EEC-9986821, the "Ministerio de Economía y Competitividad" of Spain under Projects CSD2008-00068 (TERASENSE) and TEC2011-24492/TEC (iSCAT), and by the Xunta de Galicia under Plan I2C (2011-2015) . This material is based upon work supported by the Science and Tech. Directorate, U.S. Department of Homeland Security under Award 2008-ST-061-ED0001.

Y. Álvarez and F. Las-Heras are with the Area of Signal Theory and Communications, Department of Electrical Engineering, Universidad de Oviedo, E-33203, Gijón, Spain (e-mail: yalopez@tsc.uniovi.es; flasheras@tsc.uniovi. es).

B. Gonzalez-Valdes is with the ALERT Center, Northeastern University, Boston, MA 02115 USA, and also with the Universidad de Vigo, E-36310 Vigo, Spain (e-mail: bgonzale@ece.neu.edu).

Y. R. Vaqueiro, S. Mantzavinos, J. Á. Martínez-Lorenzo, and C. M. Rappaport are with the ALERT Center, Northeastern University, Boston, MA 02115 USA (e-mail: rodriguezvaqueiro.y@husky.neu.edu; smantzavinos@gmail.com; jmartine@ece.neu.edu; rappapor@ece.neu.edu).

Digital Object Identifier 10.1109/TMTT.2014.2332307

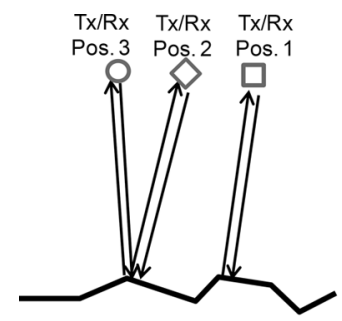

Monostatic radar

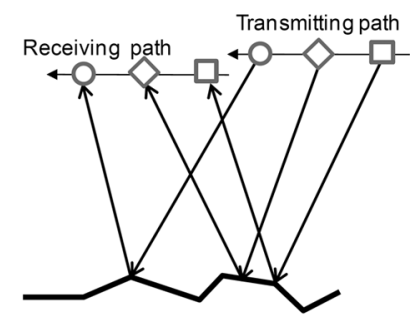

Bistatic radar

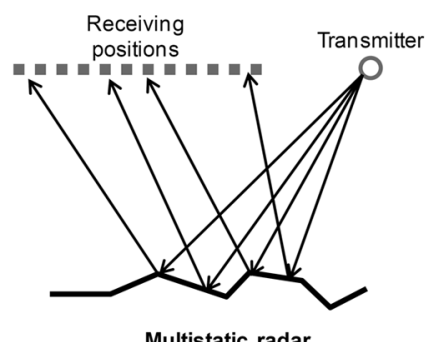

Multistatic radar

Fig. 1. Monostatic, bistatic, and multistatic radar configuration.

mentioned methods, calculation time can be a limitation. In order to overcome this issue, Fourier imaging techniques are well suited to provide a fast SAR imaging method.

In the field of monostatic radar systems, SAR imaging is mostly implemented by using fast Fourier transform (FFT) algorithms. Formulations for planar and cylindrical imaging systems, devoted for whole human body imaging, have been presented in [3], [13], and [14].

Monostatic imaging system limitations are mainly related with the appearance of reconstruction artifacts such as dihedral effects like the one between the legs in [3, Fig. 14], where the multiple reflections of the wave on the inside of each leg create an erroneous bright line between the legs.

Therefore, bistatic [15], [16] or multistatic systems [10], [17] are better suited for imaging scattering objects with sudden profile variations. Fig. 1 illustrates this fact depicting the propagation directions of incident and scattered millimeter waves for a particular geometry under test. For the monostatic case, the main contribution of the scattered field will come from the part of the geometry whose normal is opposite to the incident direction, and other parts of the geometry may not be reconstructed. This effect can be reduced in the case of bistatic or multistatic configurations.

Performing bistatic SAR imaging is more challenging than the monostatic configuration due to the need of considering the different trajectories (different angular directions) of the incident and reflected electric field. Recently, fast and efficient 
algorithms for bistatic SAR imaging have been explored: a fast back-projection algorithm, reducing computational cost to the same order as that of direct Fourier reconstruction is presented in [18]. Bistatic imaging FFT-based formulation using Fourier decomposition of spherical waves is described in [16, Sec. III]. Halcrow [19] describes a nonlinear $k$-space mapping method for SAR Fourier imaging for both monostatic and bistatic systems. Others [20] and [21] introduce Fourier-based formulation with equivalent currents for forward and inverse scattering problems with a bistatic formulation.

Multistatic radar systems can be understood as a particular case of bistatic systems: one single transmitter and multiple receiving positions (or vice versa, taking advantage of the reciprocity theorem), as shown in Fig. 1. The FFT-based imaging formulation for this kind of systems has been mainly based on monostatic equivalent approaches, as described in $[16$, Sec. IV-A]: a phase correction term is introduced to compensate for differences between monostatic and multistatic. However, these approaches have a limited range of validity, making them unsuitable for imaging electrically large objects.

A multistatic FFT-based formulation, derived from the monostatic case described in Sheen [3], is presented in this contribution. Incident field compensation is based on plane-wave illuminations.

For spherical wave-like incidence, this paper uses an approach based on multiple plane waves and then combines the results to form a single image.

Multistatic FFT-based imaging requires using multidimensional interpolation techniques which map the data in the $k$-space from a nonuniform grid into a uniform one. To overcome the issue of computational cost, the $k$-space is split into several regions where multidimensional interpolation is applied.

The main concern of the presented method is the moderate computational cost due to the following reasons: 1) spherical wave approximation using multiple plane waves and 2) multidimensional interpolation. Both are fully parallelizable, thus being well suited to take advantage of multicore platforms or graphics processing units (GPUs) [22].

The paper is organized as follows. Section II describes the multistatic Fourier-based imaging formulation, discusses the limitations of multidimensional $k$-space interpolation and spherical wave illumination, and proposes solutions. A two-dimensional (2-D) simulation-based method validation is presented in Section III, analyzing calculation time and accuracy when compared to traditional SAR backpropagation imaging. Section IV is devoted to three-dimensional (3-D) validation using radar measurements, and Section $\mathrm{V}$ makes use of 3-D simulation-based results to highlight incident field compensation performance.

\section{Multistatic Fourier IMAging Method}

\section{A. Formulation}

Here, the Fourier-based imaging formulation for a multistatic configuration is presented. For the sake of simplicity, a 2-D imaging formulation, involving range ( $z$-axis) and cross-range ( $x$-axis) will be described. Derivation of the relationship be- tween the spatial and the spectral domain has already been widely described in [3].

The scattered field $E_{\text {scatt }}(f, x)$ acquired in a linear observation domain placed along $x$-axis, for a certain frequency range $f$ can be represented in the spectral domain $k_{\mathrm{x}}$ by taking the Fourier transform in the cross-range dimension

$$
\tilde{E}_{\text {scatt }}\left(f, k_{x}\right)=\int_{x} E_{\text {scatt }}(f, x) e^{-j k_{x} x} d x
$$

with the $k_{\mathrm{x}}$ domain being defined from the aperture size and the sampling rate. Next, the field in the spectral domain is translated to the imaging domain by applying a phase shift equal to $k_{\mathrm{z}} Z_{0}$ :

$$
\tilde{E}_{\text {trans }}\left(f, k_{x}\right)=\tilde{E}_{\text {scatt }}\left(f, k_{x}\right) e^{+j k_{z} Z_{0}}
$$

where $Z_{0}$ is the distance in range from the observation domain $z$ to the imaging domain, which is assumed to be centered at $\left(z^{\prime}, x^{\prime}\right)=(0,0) . k_{\mathrm{z}}$ is defined as

$$
k_{z}=\sqrt{k^{2}-k_{x}^{2}} .
$$

Next, the incident field is specified [21]. Assuming planewave illumination

$$
\tilde{E}_{\text {shift }}\left(f, k_{x}\right)=\tilde{E}_{\text {trans }}\left(f, k_{x}\right) e^{+j k_{i n c}\left(x_{\text {inc }} \sin (\alpha)+z_{\text {inc }} \cos (\alpha)\right)}
$$

where $k_{\text {inc }}=k=2 \pi f / c$ and $\left(z_{\text {inc }}, x_{\text {inc }}\right)$ denotes the position of the transmitter. The incidence angle $\alpha$ is defined relative to the $z$-axis (see Figs. 2 or 7). To recover the image centered in the imaging domain $\left(z^{\prime}, x^{\prime}\right)$, the spectral domain is redefined as follows:

$$
k_{z}{ }^{\prime}=k_{z}+k_{z, \text { inc }} \quad k_{x}{ }^{\prime}=k_{x}+k_{x, \text { inc }}
$$

where $k_{\mathrm{x}, \text { inc }}=k_{\text {inc }} \sin (\alpha), k_{\mathrm{z} \text {,inc }}=k_{\text {inc }} \cos (\alpha)$. This spectral domain shift due to the incident field angle $\alpha$ is depicted in Fig. 2: the left plot represents the spectral domain shift for $\alpha=0$, and the right plot represents the spectral domain shift for a generic $\alpha>0$. Thus, the incident field $\tilde{E}_{\text {shift }}\left(f, k_{x}\right)$ has to be interpolated into a regular grid $k_{\mathrm{z}}{ }^{\prime}=n \Delta k_{\mathrm{z}}, k_{\mathrm{x}}{ }^{\prime}=m \Delta k_{\mathrm{x}}$. This step is the most time-consuming operation in Fourier-based imaging methods. For multistatic systems, the interpolation step becomes even more challenging as the $\left(f, k_{\mathrm{x}}\right)$ grid is not equispaced in $k_{\mathrm{x}}$ for oblique incidence, that is, $k_{\mathrm{x}, \text { inc }} \neq 0$ (see Fig. 2). The interpolation operation is defined as

$$
\tilde{E}_{\text {shift,int }}\left(k_{z}{ }^{\prime}, k_{x}{ }^{\prime}\right)=F\left\{\tilde{E}_{\text {shift }}\left(f, k_{x}\right), f \rightarrow k_{z}{ }^{\prime}, k_{x} \rightarrow k_{x}{ }^{\prime}\right\} \text {. }
$$

$F$ is the interpolation operator (e.g., linear interpolation). Finally, a 2-D inverse Fourier transform is applied to recover the reflectivity $\rho$ in the $z^{\prime}, x^{\prime}$ domain:

$\rho\left(z^{\prime}, x^{\prime}\right)=\iint_{k_{z}{ }^{\prime}} \int_{k_{x}{ }^{\prime}} \tilde{E}_{\text {shift }, \text { int }}\left(k_{z}{ }^{\prime}, k_{z}{ }^{\prime}\right) e^{+j k_{x}{ }^{\prime} x^{\prime}} e^{+j k_{x}{ }^{\prime} z^{\prime}} d k_{x}{ }^{\prime} d k_{z}{ }^{\prime}$.

\section{B. $k$-Space Interpolation}

As mentioned before, the multistatic formulation has the drawback of nonuniform grid spacing in $k_{\mathrm{x}}$ for oblique incidence, thus requiring 2-D interpolation techniques, which increases the computational complexity. 

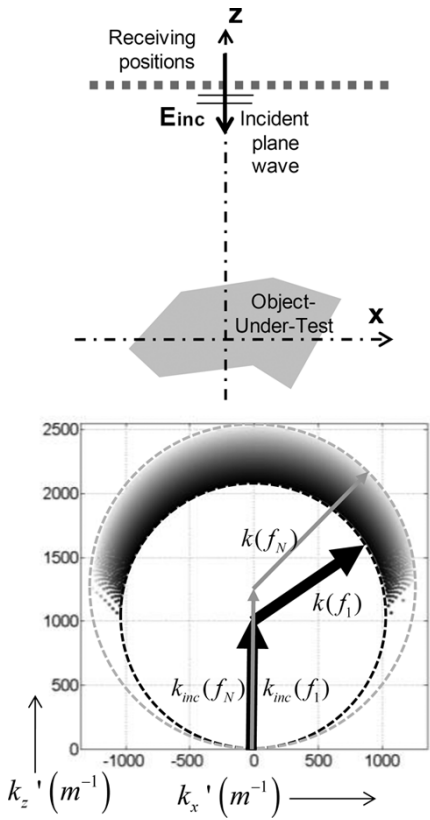

(a)
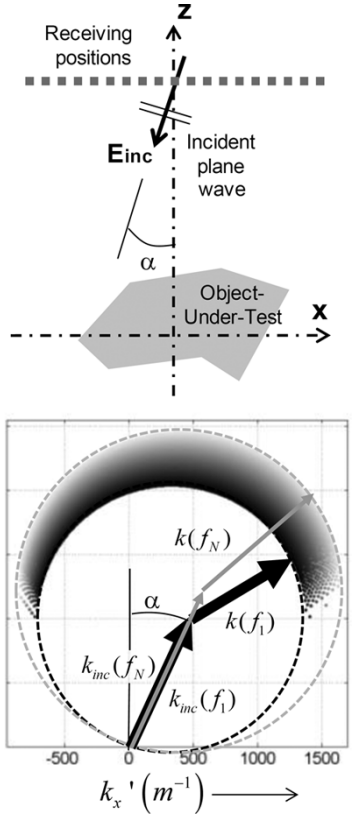

(b)

Fig. 2. Example of spectral domain for a multistatic configuration. (a) Normal incidence and (b) oblique incidence with an incidence angle $\alpha$. Field is acquired in a frequency band ranging from $f_{1}$ (dark gray) to $f_{N}$ (light gray). Points having the same frequency, $f_{\mathrm{n}}$, are depicted with the same shading.

To overcome this limitation, a strategy based on $k$-space partitioning is proposed. The idea is to divide the $\left(k_{\mathrm{z}}{ }^{\prime}, k_{\mathrm{x}}{ }^{\prime}\right)$-space domain into several subdomains. For every subdomain, the method checks if there are any $\tilde{E}_{\text {shift }}\left(f, k_{x}\right)$ values inside the subdomain. If not, $\tilde{E}_{\text {shift,int }}\left(k_{z}{ }^{\prime}, k_{x}{ }^{\prime}\right)$ is set to zero in this subdomain. Otherwise, interpolation is done using the nonzero field samples.

Thus, the smaller the subdomains, the better fitting to the spectral domain region having $\tilde{E}_{\text {shift }}\left(f, k_{x}\right) \neq 0$. This idea is depicted in Fig. 3: $\left(k_{\mathrm{z}}{ }^{\prime}, k_{\mathrm{x}}{ }^{\prime}\right)$-space is divided into $2 \times 2$ subdomains [Fig. 3(a)], $6 \times 6$ [Fig. 3(b)] and $11 \times 11$ [Fig. 3(c)]. It can be observed that: 1) for the case of $6 \times 6$ subdomains, only 28 (77\%) contain data different from zero and 2) for the case of $11 \times 11,84$ out of 121 have information (that is, $69 \%$ ).

In addition, it is noticed that only one portion of the $\left(k_{\mathrm{z}}{ }^{\prime}, k_{\mathrm{x}}{ }^{\prime}\right)$-space is considered for partitioning and interpolation. This region is defined as the minimum rectangle having data with amplitude $\geq C \mathrm{~dB}$ with respect to the $\tilde{E}_{\text {shift }}\left(f, k_{x}\right)$ maximum (e.g., $C=-40 \mathrm{~dB}$ ).

The advantage of this interpolation technique is that it can be easily parallelized, as every subdomain can be processed independently. Thus, several threads can be launched in parallel for performing interpolation operations. As the subdomains do not overlap, every thread can save the interpolated field $\tilde{E}_{\text {shift }, \text { int }}\left(k_{z}{ }^{\prime}, k_{x}{ }^{\prime}\right)$ independently.

Interpolation can be done faster by precalculating the interpolation operator $F$. This operator is a function of the geometry and the operating frequency band of the imaging system. Thus, for a given imaging system, operator $F$ can be calculated once and then applied to every new set of measured data.
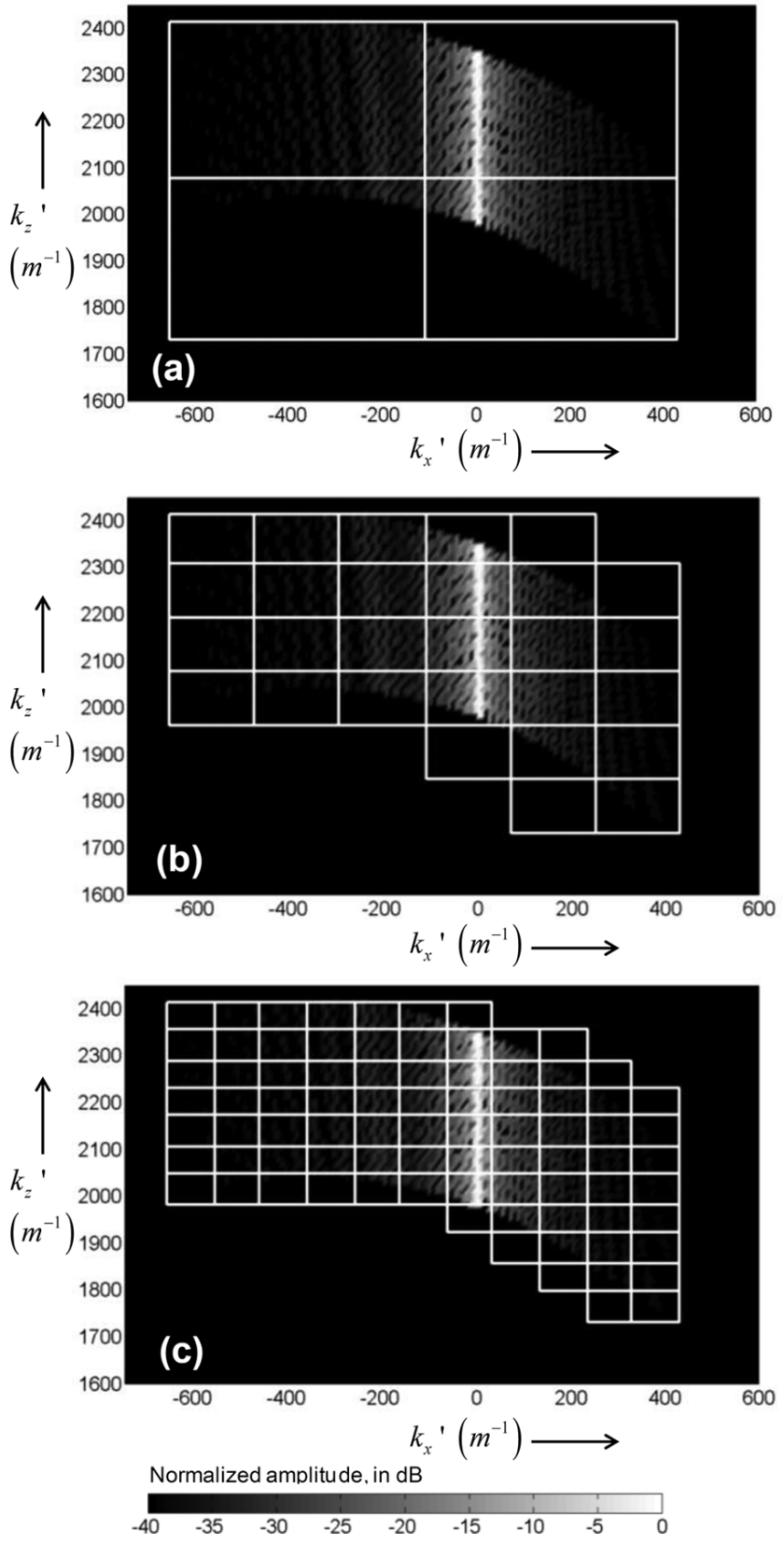

Fig. 3. Spectral domain and interpolated field $\tilde{E}_{\text {shift }, \text { int }}\left(k_{z}{ }^{\prime}, k_{x}{ }^{\prime}\right)$ (normalized amplitude, in $\mathrm{dB}$ ). Interpolation subdomains (white rectangles). (a) $2 \times 2$. (b) $6 \times 6$. (c) $11 \times 11$.

\section{Point Source Illumination}

The FFT algorithm for multistatic radar systems makes two assumptions: 1) imaging domain is centered at the origin of the coordinate system and 2) the incident field is a plane wave.

These assumptions limit the scope of application for point source-like transmitters placed in the near-field region of the imaged object. This approach will introduce a phase error in the recovered image proportional to the distance between the origin of the coordinate system and the point where the image is recovered (i.e., the difference between planar and spherical phase fronts). Thus, only the origin of the coordinate system will have zero phase error. The phase error due to the plane-wave approach is shown conceptually in Fig. 4. 


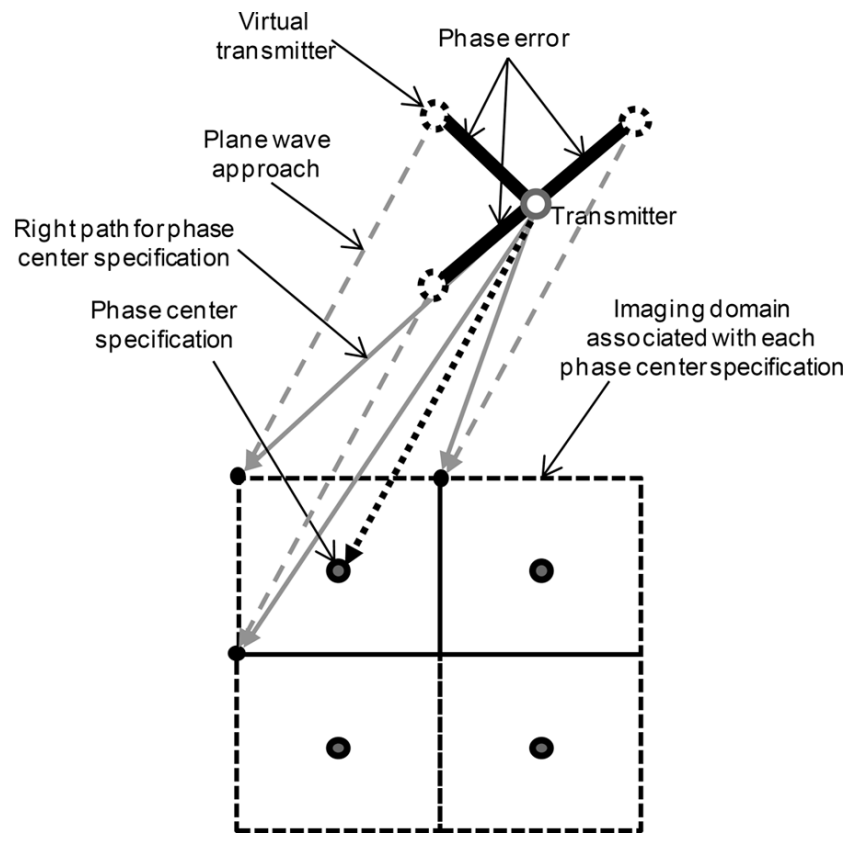

Fig. 4. Phase center specification and phase error for those points belonging to the same imaging domain.

Spherical wave expansion in terms of plane waves has been proposed in [16], introducing several approximations to simplify the formulation and defining the maximum imaging domain size where applicable.

With regard to the imaging domain size, in this contribution it is proposed to divide the imaging domain into several regions (see Fig. 4). The center point of each region is identified as phase center specification $\left(z_{C_{p}}, x_{C_{p}}\right)$. Imaging is done independently for every region, locally compensating for the incident field using a plane wave travelling from the transmitter to the phase center specification, as shown in Fig. 5. Consideration of a single plane wave allows reducing calculation time.

Several issues must be addressed in the formulation described in Section II-A. First, the scattered field observation domain must be centered in cross-range $x$ with respect to the $x$-coordinate of the phase-center specification $x_{C_{p}}$. As indicated in [16], displacement in the spatial domain is equivalent to introduce a phase shift in $k$-space

$$
\tilde{E}_{\text {scatt,cen }}\left(f, k_{x}\right)=\tilde{E}_{\text {scatt }}\left(f, k_{x}\right) e^{+j k_{x} x_{C_{p}}} .
$$

Displacement in the $z$-axis direction, which corresponds again to a phase shift in $k$-space, has to be updated taking into account the $z$-coordinate of the phase center specification $z_{C_{p}}$, as follows:

$$
\tilde{E}_{\text {trans }}\left(f, k_{x}\right)=\tilde{E}_{\text {scatt,cen }}\left(f, k_{x}\right) e^{+j k_{z}\left(Z_{0}-z_{C_{p}}\right)} .
$$

Thus, for incident field phase-center specification, we have

$$
\tilde{E}_{\text {shift }}\left(f, k_{x}\right)=\tilde{E}_{\text {trans }}\left(f, k_{x}\right) e^{+j k_{i n c}^{C_{p}} D_{\text {inc }}^{C_{p}}}
$$

where the terms $D_{\text {inc }}^{C_{p}}$ (the distance from the transmitter to the $p$ th phase center specification) and $k_{\text {inc }}^{C_{p}}$ are defined as (Fig. 5)

$$
D_{\text {inc }}^{C_{p}}=\sqrt{\left(x_{\text {inc }}-x_{C_{p}}\right)^{2}+\left(z_{\text {inc }}-z_{C_{p}}\right)^{2}} \quad k_{\text {inc }}^{C_{p}}=k .
$$
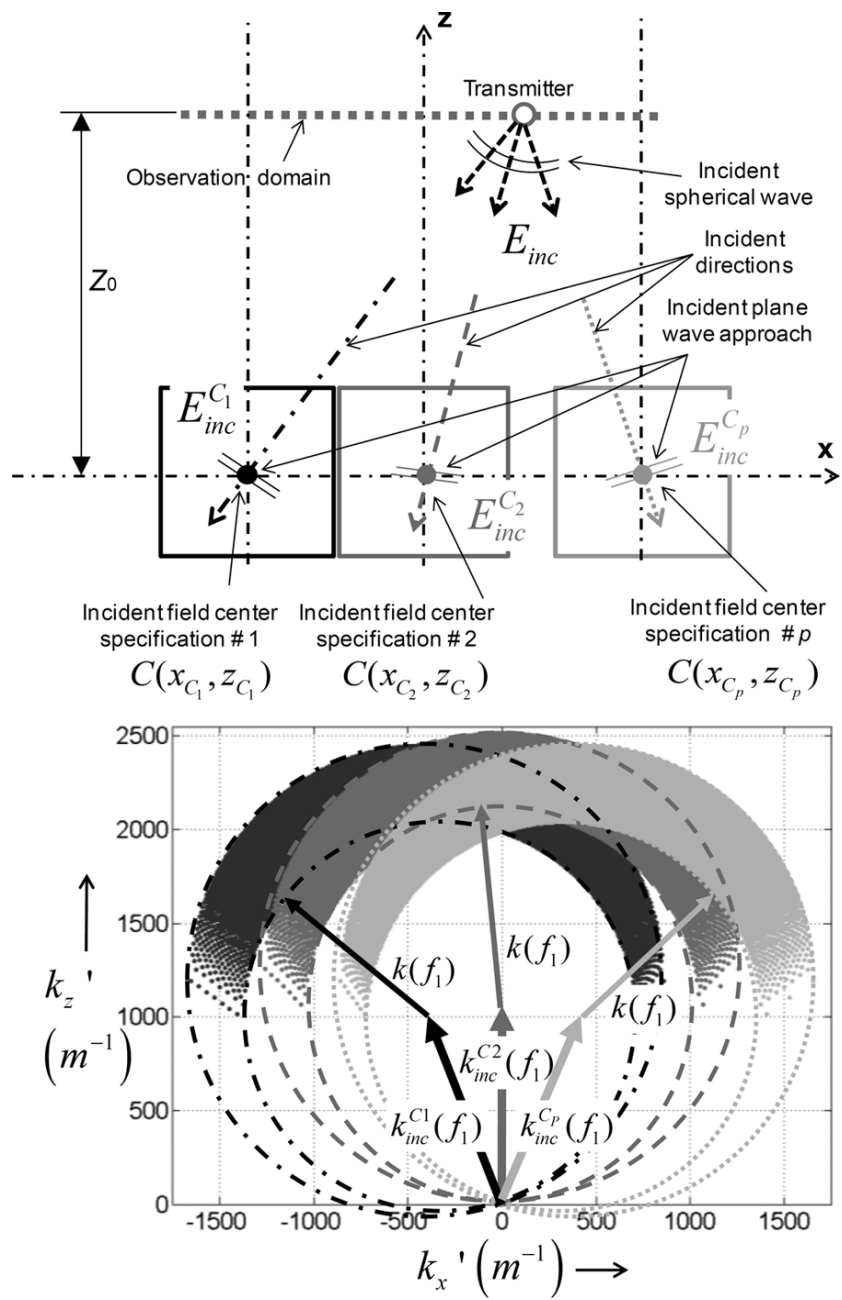

Fig. 5. Incident field compensation using several phase-center specification. A spherical incident wave is approximated as a combination of $p$-plane waves with different incident directions. Top: depiction of the imaging setup. Bottom: $k$-space domain displacement for every phase center specification (circles with the same gray shading).

The next step is to displace the resulting reflectivity image from the origin of the coordinate system to the phase center specification. This shifting operation $S$, is defined as

$$
\begin{aligned}
& \rho\left(z^{\prime}-z_{C_{p}}, x^{\prime}-x_{C_{p}}\right) \\
& \quad=S\left\{\rho\left(z^{\prime}, x^{\prime}\right), z^{\prime} \rightarrow\left(z^{\prime}-z_{C_{p}}\right), x \rightarrow\left(x^{\prime}-x_{C_{p}}\right)\right\} .
\end{aligned}
$$

Finally, reflectivity images recovered for every phase-center specification are combined in a single image. Among several methods for image combination, a simple windowing technique is chosen: points further than a certain distance $L_{\mathrm{z}}, L_{\mathrm{x}}$ from the phase center specification are discarded as follows:

$$
\begin{aligned}
& \rho_{W}\left(z^{\prime}-z_{C_{p}}, x^{\prime}-x_{C_{p}}\right)=W\left\{\rho\left(z^{\prime}-z_{C_{p}}, x^{\prime}-x_{C_{p}}\right)\right\} \\
& W=\left\{\begin{array}{c}
\text { if }\left(\left|z^{\prime}\right|>L_{z} \cup\left|x^{\prime}\right|>L_{x}\right), \text { then } \\
\rho_{W}\left(z^{\prime}-z_{C_{p}}, x^{\prime}-x_{C_{p}}\right)=0 \\
\text { otherwise, } \\
\rho_{W}\left(z^{\prime}-z_{C_{p}}, x^{\prime}-x_{C_{p}}\right)=\rho\left(z^{\prime}-z_{C_{p}}, x^{\prime}-x_{C_{p}}\right) .
\end{array}\right.
\end{aligned}
$$


If the phase-center specifications form a rectangular $z^{\prime}, x^{\prime}$ grid, then $L_{\mathrm{z}}$ and $L_{\mathrm{x}}$ are half the spacing between phase centers. Now that the phase0center specification terms have been defined, it is possible to quantitatively define the phase error $\left(\varepsilon_{\phi}\right)$ as the difference between an incident spherical wave and the plane-wave approach

$$
\begin{aligned}
\varepsilon_{\phi} & =\langle\phi, \mathrm{SPH}\rangle-\langle\phi, \mathrm{PW}\rangle \\
\langle\phi, \mathrm{SPH}\rangle & =k \sqrt{\left(x^{\prime}-x_{\text {inc }}\right)^{2}+\left(z^{\prime}-z_{\text {inc }}\right)^{2}} \\
\langle\phi, \mathrm{PW}\rangle & =k\left(x_{i n c}-x_{C_{p}}\right) \sin (\alpha)+k\left(z_{\text {inc }}-z_{C_{p}}\right) \cos (\alpha)
\end{aligned}
$$

which is represented in Fig. 4 by thick segments.

Incident field specification based on multiple phase centers has the drawback of multiplying the Fourier imaging calculation time by the number of centers considered. Fortunately, the problem associated with every phase center can be processed independently, thus allowing parallelization. Note that, for every parallel problem, interpolation can still be done in parallel. Thus, ideally, assuming there are sufficient available parallel threads, the calculation time of the Fourier-based method is limited by the interpolation time of the $k$-space domain with highest number of nonzero elements $\left(\tilde{E}_{\text {shift }}\left(f, k_{x}\right) \neq 0\right)$.

The proposed Fourier-based imaging method for multistatic systems is summarized in the flow chart of Fig. 6.

\section{2-D Simulation Results}

First, the Fourier-based imaging method is tested with 2-D simulation-based examples. The object-under-test (OUT) is a metallic plate $50 \mathrm{~cm}$ long, centered at the origin of the coordinate system. The working frequency band is $50-60 \mathrm{GHz}$, sampled every $500 \mathrm{MHz}$. An array of receivers $L_{\text {array }}=80 \mathrm{~cm}$ long, is placed at $Z_{0}=60 \mathrm{~cm}$ with a sampling rate of $1 \mathrm{~mm}$ $(\lambda / 5$ at $60 \mathrm{GHz})$. Thus, range and cross-range resolution are 15 and $4.5 \mathrm{~mm}$, respectively. Cross-range resolution is calculated as $\lambda Z_{0} / L_{\text {array }}$, with $\lambda$ evaluated at $50 \mathrm{GHz}$. No windowing function is applied either in range or cross range. The scattered field is simulated using a 2-D method-of-moments MATLAB code that implements electric field integral equations for TM polarization [23].

The simulation examples were run in a laptop equipped with Intel Core i5 CPU M560 @ 2.67 GHz, with 4 GB RAM. For benchmarking purposes, Fourier and SAR backpropagation methods were run in single-core mode. SAR backpropagation [9], [10] is given by

$$
\rho\left(z^{\prime}, x^{\prime}\right)=\sum_{f} \sum_{x}\left\{\begin{array}{l}
E_{\text {scatt }}(f, x) \\
\cdot e^{+j k f\left(\left(x-x^{\prime}\right)^{2}+\left(Z_{0}-z^{\prime}\right)^{2}\right)^{1 / 2}} \\
\cdot e^{+j k f\left(\left(x_{i n c}-x^{\prime}\right)^{2}+\left(z_{i n c}-z^{\prime}\right)\right)^{1 / 2}}
\end{array}\right\} d f d x .
$$

Both SAR and FFT equations are coded using MATLAB. The motivation for using this platform is the ease of code debugging, at the expense of increased calculation time. To ensure proper calculation time comparison, MATLAB optimized routines, such as indexing, interpolation, and FFT functions, have been used in both SAR backpropagation and FFT codes.

Concerning memory consumption, neither FFT nor SAR require more than $1 \mathrm{~GB}$ of RAM in any of the examples shown

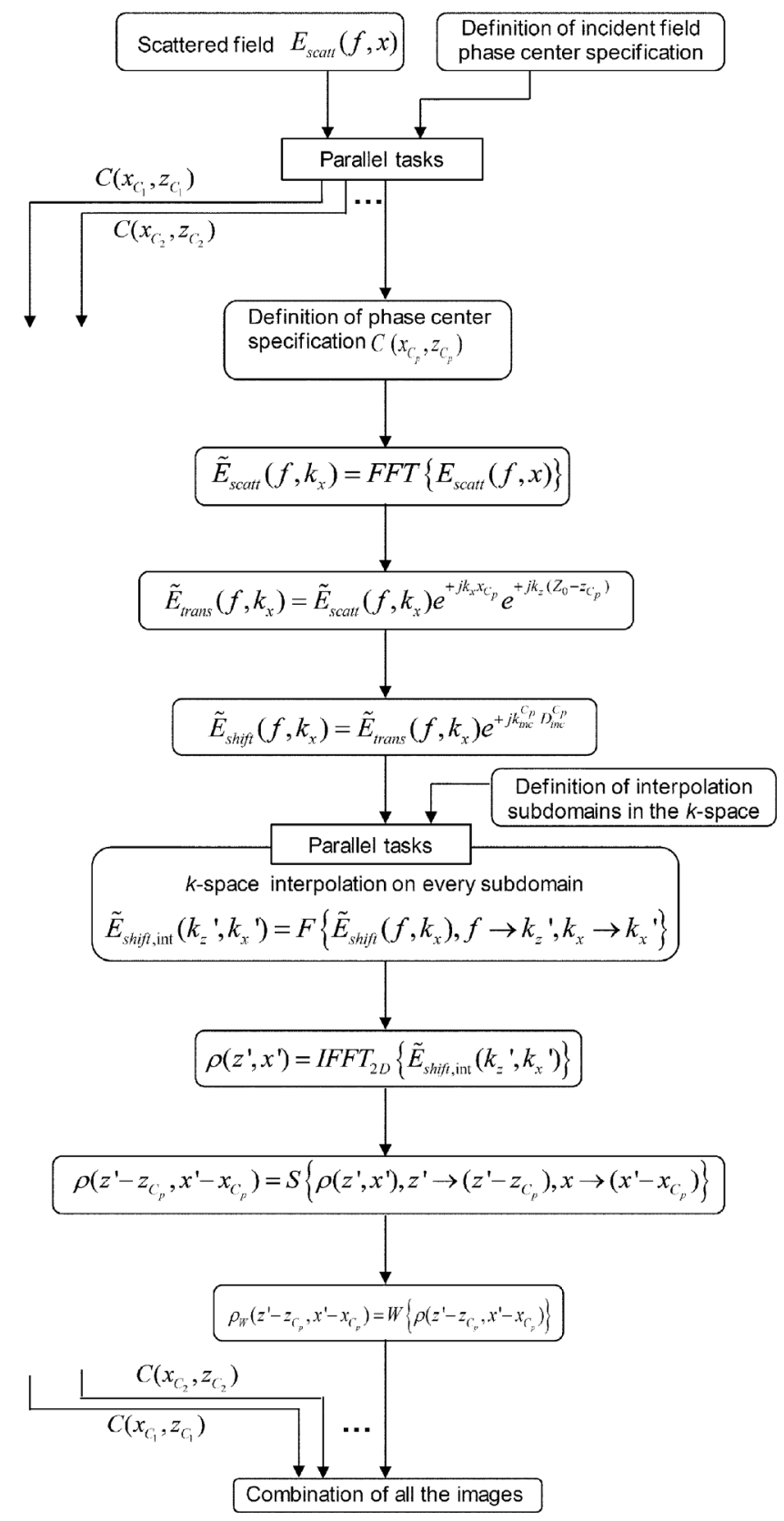

Fig. 6. Block diagram of the Fourier imaging algorithm for multistatic radar systems.

in this paper, so this parameter is not included in the computational cost analysis.

\section{A. Plane-Wave Illumination}

The first analyzed case makes use of a normal illumination of the OUT using a plane wave propagating towards $-z$, as shown in Fig. 7(a) (with $\alpha=0^{\circ}$ ). In this case, as a plane wave is used for OUT illumination, Fourier imaging is accurate without requiring multiple phase-center specifications different than the origin of the coordinate system.

Recovered reflectivity is shown in Fig. 8, where traditional SAR imaging is used for benchmarking. It can be noticed that the differences are negligible, with a root mean square error (RMSE) of $-39.4 \mathrm{~dB}$. In this case, Fourier imaging is as exact 


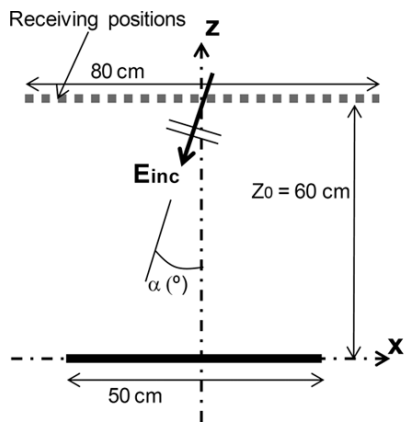

(a)

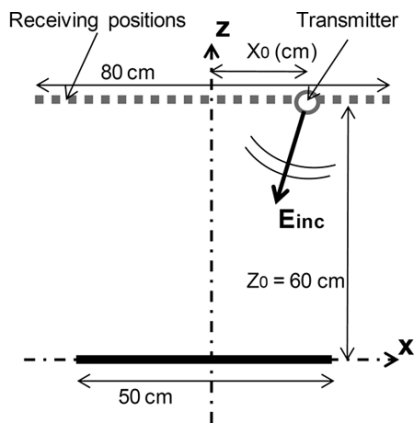

(b)
Fig. 7. 2-D simulation-based test cases. (a) Plane-wave illumination. (b) Point source transmitter (spherical illumination).
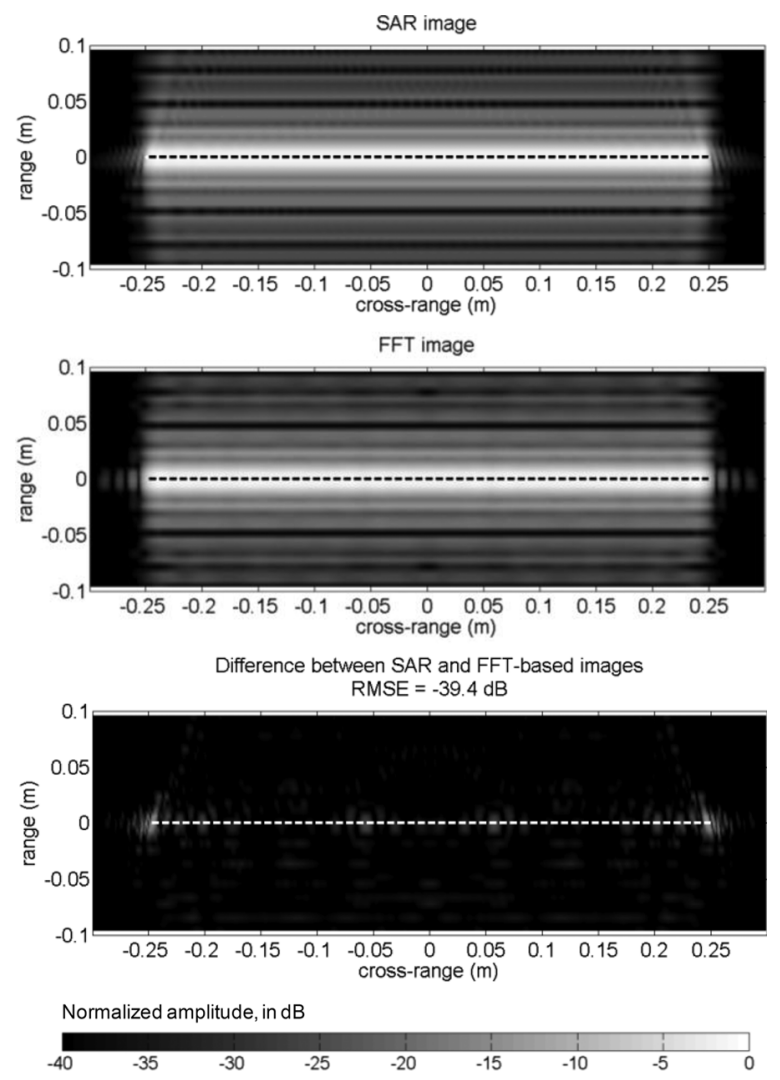

Fig. 8. Recovered reflectivity for a plate. Incident field: plane wave with normal incidence $\left(\alpha=0^{\circ}\right)$. Top: SAR backpropagation imaging. Center: Fourier imaging method. Bottom: amplitude difference between SAR and Fourier. True metallic plate profile is represented with a dashed line.

as SAR backpropagation because the incident field is a plane wave.

SAR backpropagation processing takes $35 \mathrm{~s}$, whereas Fourier imaging method takes less than $1 \mathrm{~s}$. Interpolation time and imaging accuracy as a function of $k$-space partitioning is shown in Table I. It can be noticed that the interpolation time does not decrease for a larger number of subdomains. This is due to the time needed to create the subdomains. Regarding accuracy, variation in the number of subdomains modifies the RMSE less than $0.2 \mathrm{~dB}$.

The same configuration is tested when the OUT is illuminated using a plane wave with $\alpha=20^{\circ}$. Results are shown in Fig. 9.
TABLE I

ANALYSIS OF ACCURACY AND CALCULATION TIME FOR DIFFERENT NUMBERS of K-Space Subdomains

\begin{tabular}{cccc}
\hline \hline $\begin{array}{c}\text { No. of } \\
\text { domains }\end{array}$ & $\begin{array}{c}\text { Domains with } \\
\text { field } \neq 0(\%)\end{array}$ & $\begin{array}{c}\text { SAR-Fourier } \\
\text { RMSE }(\mathrm{dB})\end{array}$ & $\begin{array}{c}\text { Interpolation time } \\
(\mathrm{ms})\end{array}$ \\
\hline $1 \times 1$ & 100 & -39.5 & 470 \\
$2 \times 2$ & 100 & -39.4 & 390 \\
$3 \times 3$ & 88.8 & -39.4 & 310 \\
$4 \times 4$ & 87.5 & -39.3 & 330 \\
$5 \times 5$ & 80 & -39.4 & 360 \\
$6 \times 6$ & 77.8 & -39.5 & 410 \\
$11 \times 11$ & 69.4 & -39.3 & 930 \\
\hline \hline
\end{tabular}
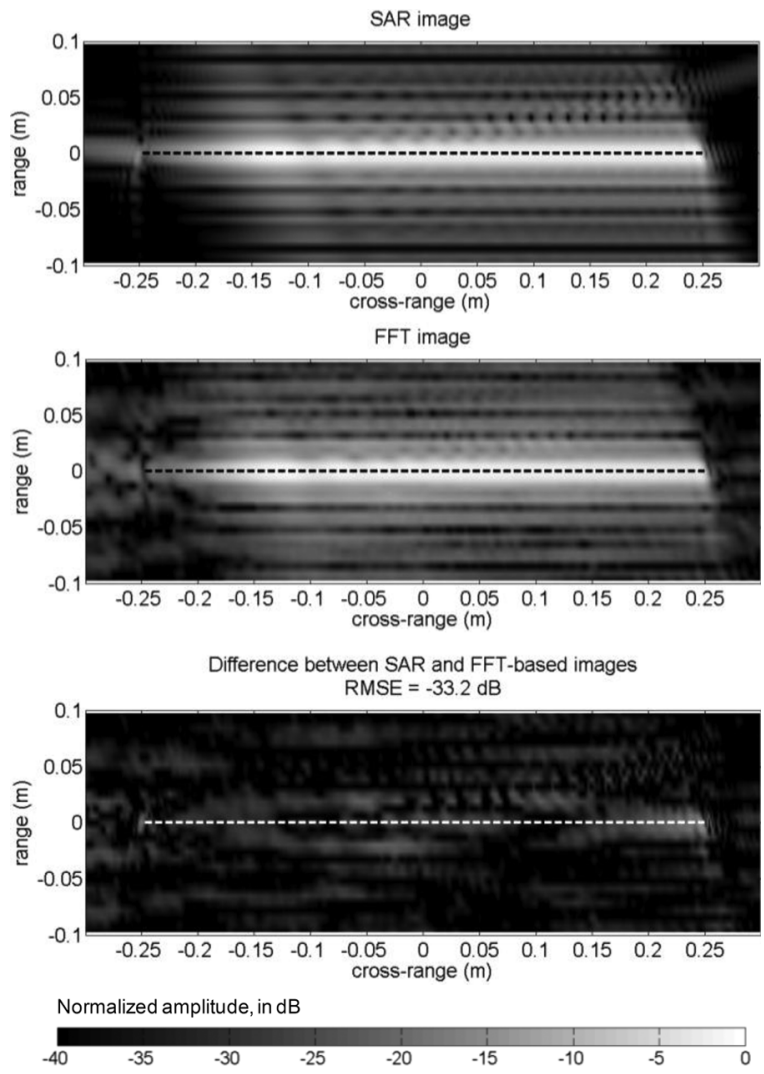

Fig. 9. Recovered reflectivity for a plate. Incident field: plane wave with oblique incidence $\left(\alpha=20^{\circ}\right)$. SAR imaging (top). Fourier method (center). Amplitude difference between SAR and Fourier (bottom). True metallic plate profile is represented with a dashed line.

In this case, the agreement between SAR and Fourier is $6 \mathrm{~dB}$ worse, with RMSE $-33.1 \mathrm{~dB}$. This occurs because $k_{\mathrm{x}}$ spacing in the $k$-space is not uniform with respect to $f$, so 2-D interpolation is less accurate than in the previous case with normal incidence, in which $k_{\mathrm{x}}$ and $k_{\mathrm{x}}{ }^{\prime}$ match.

\section{B. Point Source Illumination}

Once the Fourier imaging performance for plane-wave illumination has been demonstrated, the next step is to test point source illumination. A transmitting point source is placed at $\left(Z_{0}, X_{0}\right)=(60,0) \mathrm{cm}$ [Fig. 7(b)], plotting reflectivity recovered using a single phase center specification placed at the origin of the coordinate system in Fig. 10 (center). Compared with SAR imaging (Fig. 10, top), the plane-wave approximation of 

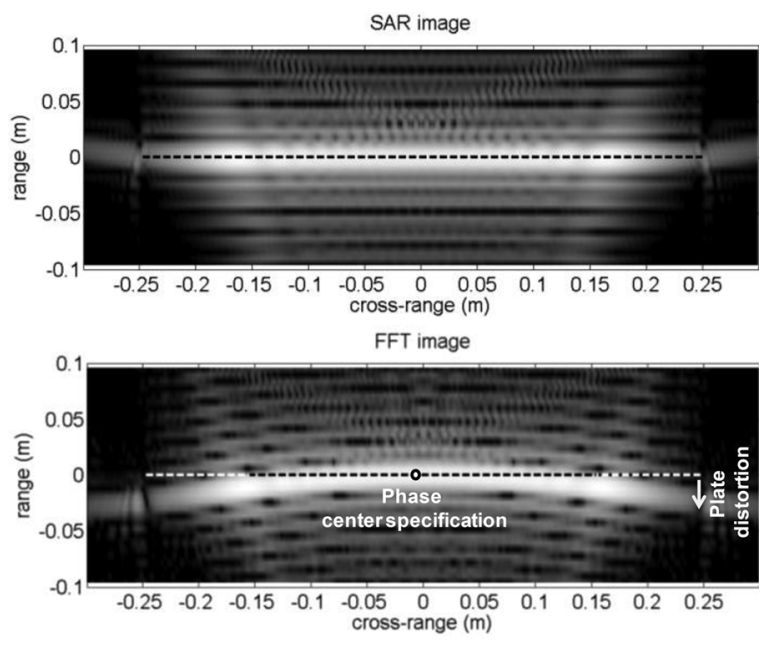

Difference between SAR and FFT-based images RMSE $=-19.4 \mathrm{~dB}$

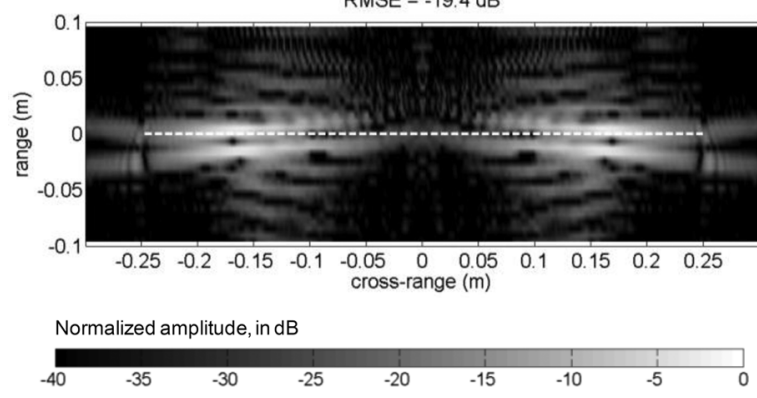

Fig. 10. Recovered reflectivity for a plate. Incident field: point source placed at $\left(Z_{0}, X_{0}\right)=(60,0) \mathrm{cm}$. Top: SAR imaging. Center: Fourier method. Bottom: amplitude difference between SAR and Fourier. True metallic plate profile is represented with a dashed line.

the point source distorts the image. Distortion increases towards the edge of the image, as depicted in Fig. 4. At the edges of the plate, the image distortion is given by

$$
\text { distortion }=\frac{1}{2}\left(\sqrt{\left(D_{\text {inc }}^{(0,0)}\right)+\left(\frac{1}{2} X_{\text {plate }}\right)^{2}}-D_{\text {inc }}^{(0,0)}\right) .
$$

$D_{\text {inc }}^{(0,0)}$ is the distance from the transmitter to the center of the plate, which is $60 \mathrm{~cm}$. $X_{\text {plate }}$ is the size of the metallic plate $(50 \mathrm{~cm})$. Thus, the distortion is $2.5 \mathrm{~cm}$ (or $5 \lambda @ 60 \mathrm{GHz}$ ), which is in agreement with the result depicted in Fig. 10.

Image distortion can be improved by considering several phase center specification in the imaging region. For this case, a rectangular grid is considered, with $x_{C_{p}}$ ranging from -25 to $25 \mathrm{~cm}$, and $z_{C_{p}}$ from -10 to $10 \mathrm{~cm}$, with $L_{\mathrm{x}}=L_{\mathrm{z}}=10 \mathrm{~cm}$; resulting in $6 \times 3=18$ phase-center specification.

The reconstructed reflectivity map is depicted in Fig. 11 (top). Compared to with Fig. 10 (center), the RMSE between SAR and Fourier improves from -19.4 to $-32.4 \mathrm{~dB}$.

Accuracy analysis and calculation time for multiple phase center specification are summarized in Table II. For all cases, $4 \times 4 k$-space interpolation subdomains are considered. The imaging domain size is $L_{\mathrm{x}}=60 \mathrm{~cm}, L_{\mathrm{z}}=20 \mathrm{~cm}$. The maximum distortion due to plane wave approximation occurs at the point $\left(z^{\prime}, x^{\prime}\right)=(10, \pm 30) \mathrm{cm}$.

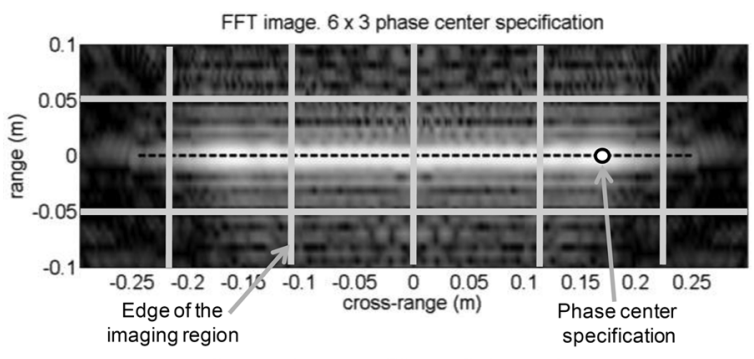

Difference between SAR and FFT-based images

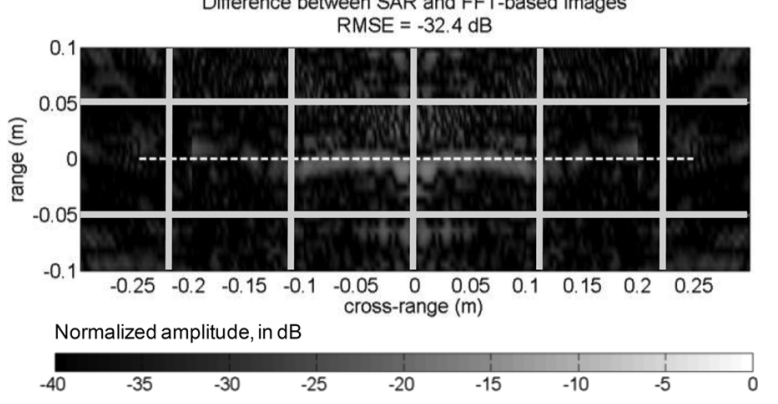

Fig. 11. Recovered reflectivity for a plate. Incident field: point source placed at $\left(Z_{0}, X_{0}\right)=(60,0) \mathrm{cm}$. Top: Fourier method with $6 \times 3$ phase-center specification. Bottom: Amplitude difference between SAR and Fourier with phase specification. Gray solid lines represent the edges of the imaging domain for every phase-center specification. True metallic plate profile is represented with a dashed line.

TABLE II

ANALYSIS OF ACCURACY AND CALCULATION TIME FOR DifFERENT NumBERS of Phase Center Specification Points

\begin{tabular}{cccccc}
\hline \hline $\begin{array}{c}\text { No. of } \\
\text { centers }\end{array}$ & $\begin{array}{c}L_{\mathrm{x}} \\
(\mathrm{cm})\end{array}$ & $\begin{array}{c}L_{\mathrm{z}} \\
(\mathrm{cm})\end{array}$ & $\begin{array}{c}\text { Maximum } \\
\text { distortion } \\
(\lambda)\end{array}$ & $\begin{array}{c}\text { SAR-FFT } \\
\text { RMSE } \\
(\mathrm{dB})\end{array}$ & $\begin{array}{c}\text { FFT overall } \\
\text { calculation } \\
\text { time }(\mathrm{ms})\end{array}$ \\
\hline $1 \times 1$ & 60 & 20 & 8.3 & -19.4 & 540 \\
$2 \times 1$ & 30 & 20 & 2.2 & -24.5 & 920 \\
$3 \times 1$ & 20 & 20 & 0.99 & -28.1 & 1290 \\
$4 \times 1$ & 15 & 20 & 0.56 & -28.9 & 1560 \\
$6 \times 2$ & 10 & 10 & 0.25 & -32.4 & 4600 \\
$12 \times 4$ & 5 & 5 & 0.06 & -32.8 & 18500 \\
\hline \hline
\end{tabular}

${ }^{1}$ Maximum distortion evaluated at $\left(z^{\prime}, x^{\prime}\right)=(10, \pm 30) \mathrm{cm}$.

As a reference, calculation time for SAR is $50 \mathrm{~s}$. In the case of Fourier imaging, for a large number of phase-center specifications, the average calculation time per center tends to less than $250 \mathrm{~ms}$.

A more complex scenario is shown in Fig. 12. The scattered field observation domain remains the same, but now the transmitter is placed at $\left(Z_{0}, X_{0}\right)=(40,-30) \mathrm{cm}$. Several arbitrary-shaped OUT are spread inside a $60 \times 60 \mathrm{~cm}$ imaging domain. The frequency range is $50-60 \mathrm{GHz}$, with $250-\mathrm{MHz}$ steps, so the unambiguity distance is $60 \mathrm{~cm}$.

For benchmarking purposes, the SAR backpropagation image is retrieved [Fig. 12(a)]. Reconstructed reflectivity using the Fourier method with phase specification at the center of the coordinate system is depicted in Fig. 12(b). The distortion introduced by approximating the incident spherical wavefront with a plane wave is clearly visible: maximum imaging distortion is $12 \lambda$ at $\left(z^{\prime}, x^{\prime}\right)=(30, \pm 30) \mathrm{cm}$. To correct this, 36 phase-center specifications are considered, with $x_{C_{p}}$ ranging from -30 to $30 \mathrm{~cm}$, and $z_{C_{p}}$ from -25 to $25 \mathrm{~cm}$, 

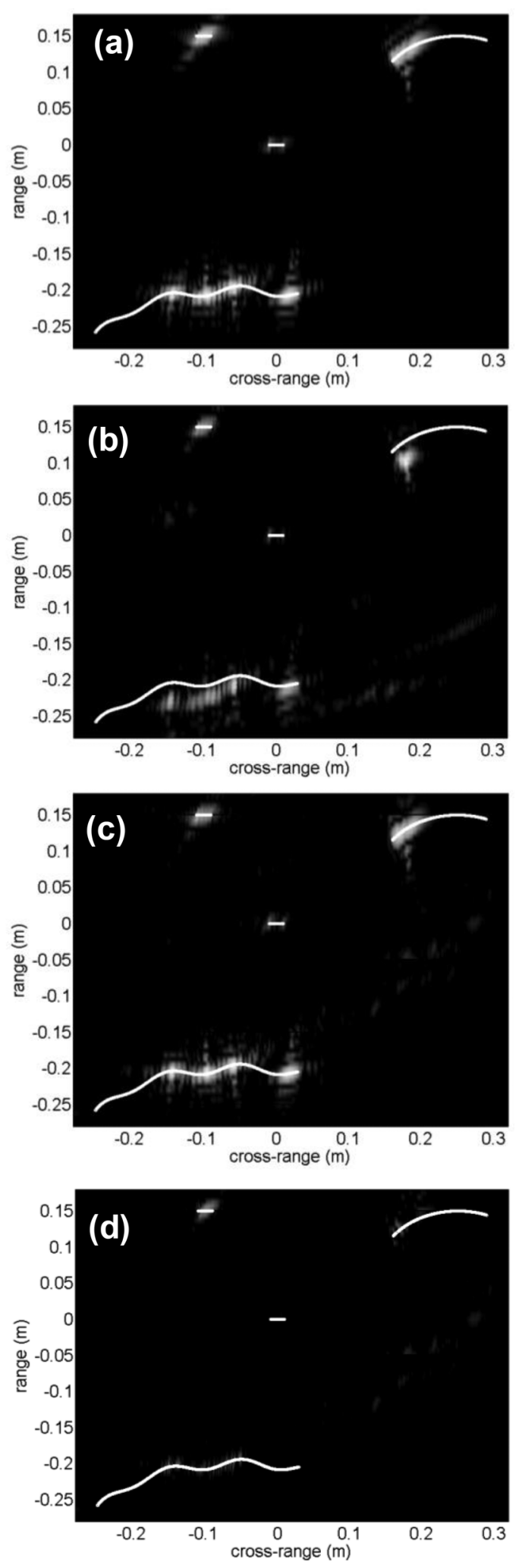

Normalized amplitude, in $\mathrm{dB}$

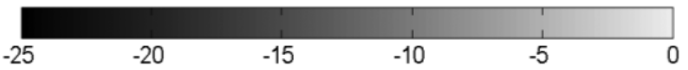

Fig. 12. Recovered reflectivity for several OUT. Incident field: point source placed at $\left(Z_{0}, X_{0}\right)=(40,-30) \mathrm{cm}$. (a) SAR imaging. (b) Fourier with phase center specification at the center of the coordinate system. (c) Fourier with 36 phase-center specification. (d) Amplitude difference between (a) and (c). Thick white lines represent the true profile of the OUT.

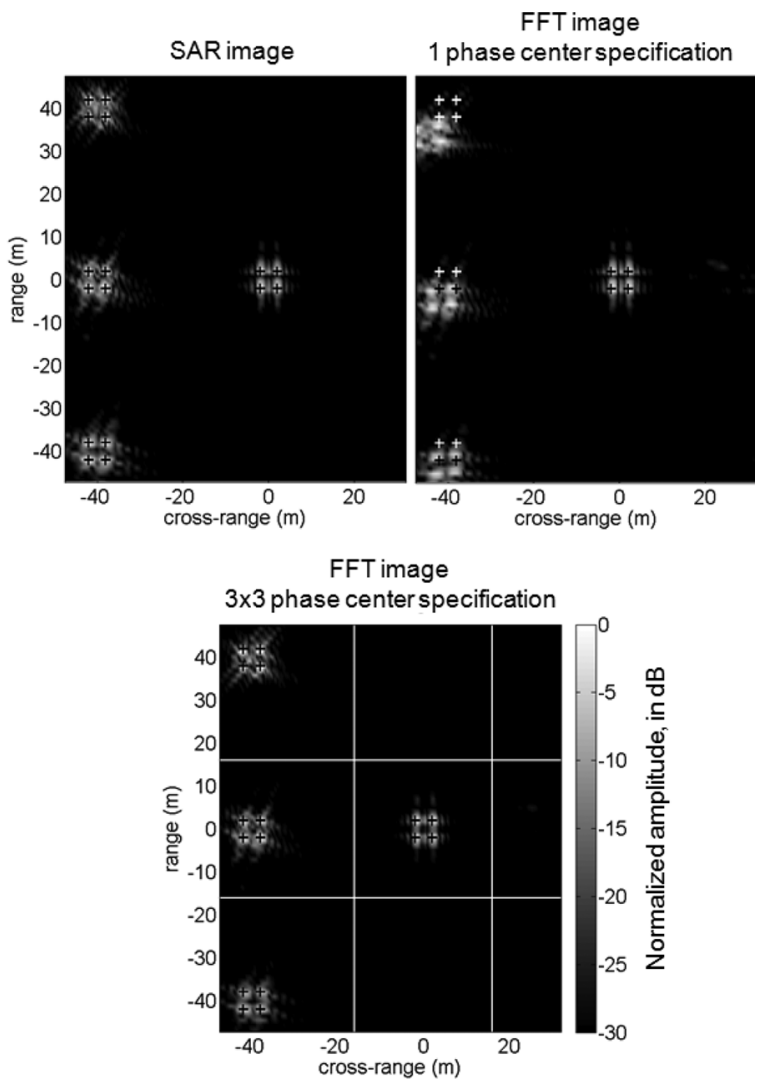

Fig. 13. Recovered reflectivity for the example presented in [16, Sec. IV-A]. SAR and FFT with $1 \times 1$ and $3 \times 3$ phase-center specification imaging results. Crosses represent the point scatterers.

with $L_{\mathrm{x}}=L_{\mathrm{z}}=10 \mathrm{~cm}$, yielding $0.4 \lambda$ maximum distortion at $\left(z^{\prime}, x^{\prime}\right)=(30, \pm 30) \mathrm{cm}$. Fig. 12(c) shows the recovered reflectivity with 36 phase centers, whereas amplitude difference with SAR is depicted in Fig. 12(d): RMSE is $-32.7 \mathrm{~dB}$.

SAR takes $420 \mathrm{~s}$ for this reconstruction. Fourier with 36 phase-center specification takes 19 s, i.e., it is 22 times faster than SAR.

\section{Comparison With an Existing Imaging Setup}

To conclude 2-D analysis, a comparison with an existing imaging setup is presented. Details of the selected example are fully described in Section IV.A of [16]. Results with SAR backpropagation and FFT with multiple phase-center specifications are depicted in Fig. 13. The use of a single phase-center specification (placed at $x_{C_{p}}=z_{C_{p}}=0 \mathrm{~m}$ ) creates a distorted image of the targets placed on the left side of the image. If $3 \times 3$ phase-center specifications are considered, correct recovery of the targets position is achieved. A summary of the main FFT parameters for different numbers of phase-center specification is shown in Table III.

Maximum image distortion is evaluated using the following expression:

$$
\begin{aligned}
\text { distortion }=\frac{1}{2}\left(\sqrt{\left(Z_{\mathrm{Tx}-\text { imaging }}\right)^{2}+}\right. & \left(\frac{1}{2} L_{x}\right)^{2} \\
& \left.-Z_{\mathrm{Tx}-\text { imaging }}\right) .
\end{aligned}
$$


TABLE III

ANALYSIS OF ACCURACY AND CALCULATION TIME FOR DifFERENT NuMBERS of PHASE-CENTER SpeCificATION POINTS

\begin{tabular}{cccccc}
\hline \hline $\begin{array}{l}\text { No. of } \\
\text { centers }\end{array}$ & $L_{\mathrm{x}}(\mathrm{m})$ & $L_{\mathrm{z}}(\mathrm{m})$ & $\begin{array}{c}\text { Maximum } \\
\text { distortion } \\
(\lambda)\end{array}$ & $\begin{array}{c}\text { SAR-FFT } \\
\text { RMSE } \\
(\mathrm{dB})\end{array}$ & $\begin{array}{c}\text { FFT overall } \\
\text { calculation } \\
\text { time }(\mathrm{s})\end{array}$ \\
\hline $1 \times 1$ & 96 & 96 & 21.8 & -25.3 & 3.8 \\
$2 \times 2$ & 48 & 48 & 6.4 & -28.7 & 9.5 \\
$3 \times 3$ & 32 & 32 & 2.9 & -34.5 & 20.6 \\
$4 \times 4$ & 24 & 24 & 1.7 & -34.7 & 37.6 \\
\hline \hline
\end{tabular}

$Z_{\text {Tx-imaging }}$ is the range distance from the transmitter to the closest limit of the imaging domain, which is $42 \mathrm{~m}$ for this example.

\section{3-D VALidation With Measurements}

Next, the Fourier-based multistatic imaging algorithm is validated with 3-D problems. This section is devoted to testing the proposed Fourier-based method on a real multistatic radar system. A general layout and a photograph of the radar are shown in Fig. 14. The working frequency band is from 72 to $74.66 \mathrm{GHz}$, sampled every $445 \mathrm{MHz}$ (range resolution, $y$-axis, is $5.6 \mathrm{~cm}$ ). The transmitting antenna is translated within $D_{\mathrm{x}}=$ $50 \mathrm{~cm}, D_{\mathrm{z}}=20 \mathrm{~cm}$ domain, placed $Y_{\mathrm{Tx}}=-1.1 \mathrm{~m}$ away from the center of the coordinate system, creating a synthetic transmitting aperture. This domain is sampled every $0.5 \lambda$ at the highest frequency $(4 \mathrm{~mm})$, resulting in $251 \times 101$ transmitting positions. Range resolution ( $y$-axis) is $56.4 \mathrm{~mm}$, and cross-range resolution is $9 \mathrm{~mm}(x$-axis $) \times 22.9 \mathrm{~mm}(z$-axis $)$. As in the previous section, no windowing function is applied. The receiver is placed at the position $(x, y, z)=(15,-134,20) \mathrm{cm}$.

The imaging domain depends on the size of the acquisition domain (or reciprocally, the size of the area within which the transmitter is moved) and the system bandwidth. For this case, the imaging region has a size of $L_{\mathrm{x}}=50 \mathrm{~cm}, L_{\mathrm{y}}=34 \mathrm{~cm}$, $L_{\mathrm{z}}=20 \mathrm{~cm}$, having $251 \times 101 \times 81$ points. For the transmitting domain aperture center, $(x, z)=(0,0) \mathrm{cm}$, and the receiver position, the imaging domain should be centered in the cross range at $(x, z)=(7.5,10) \mathrm{cm}$, i.e., in the center position in the cross range. Two metallic objects are chosen as targets (see Fig. 14).

The SAR and Fourier imaging codes were run in a workstation equipped with 12 available CPUs@3.15 GHz, with 128 GB RAM. In this case, all of the available CPUs were used. The SAR results are depicted in Fig. 15, requiring $1400 \mathrm{~s}$ of calculation time. Fourier-based results are shown in Fig. 16, taking $10 \mathrm{~s}$ of calculation (8-s interpolation tasks, 1-s FFT and IFFT operations, $1 \mathrm{~s}$ of remaining calculations), which is close to real-time capabilities. As mentioned before, interpolation operator $F$ could be reused for the same imaging setup, thus saving $8 \mathrm{~s}$.

Assuming no a priori information about the OUT is known, the phase center is set to the center position in cross-range between transmitting domain center and receiver, $(x, y, z)=(7.5,0,10) \mathrm{cm}$. Under this assumption, the RMSE between Fourier and SAR results is $-23 \mathrm{~dB}$. For the OUT centered at $(x, y, z)=(2.5,-2.5,18) \mathrm{cm}$, the phase error at this point is $1.2 \lambda$ at the highest frequency.
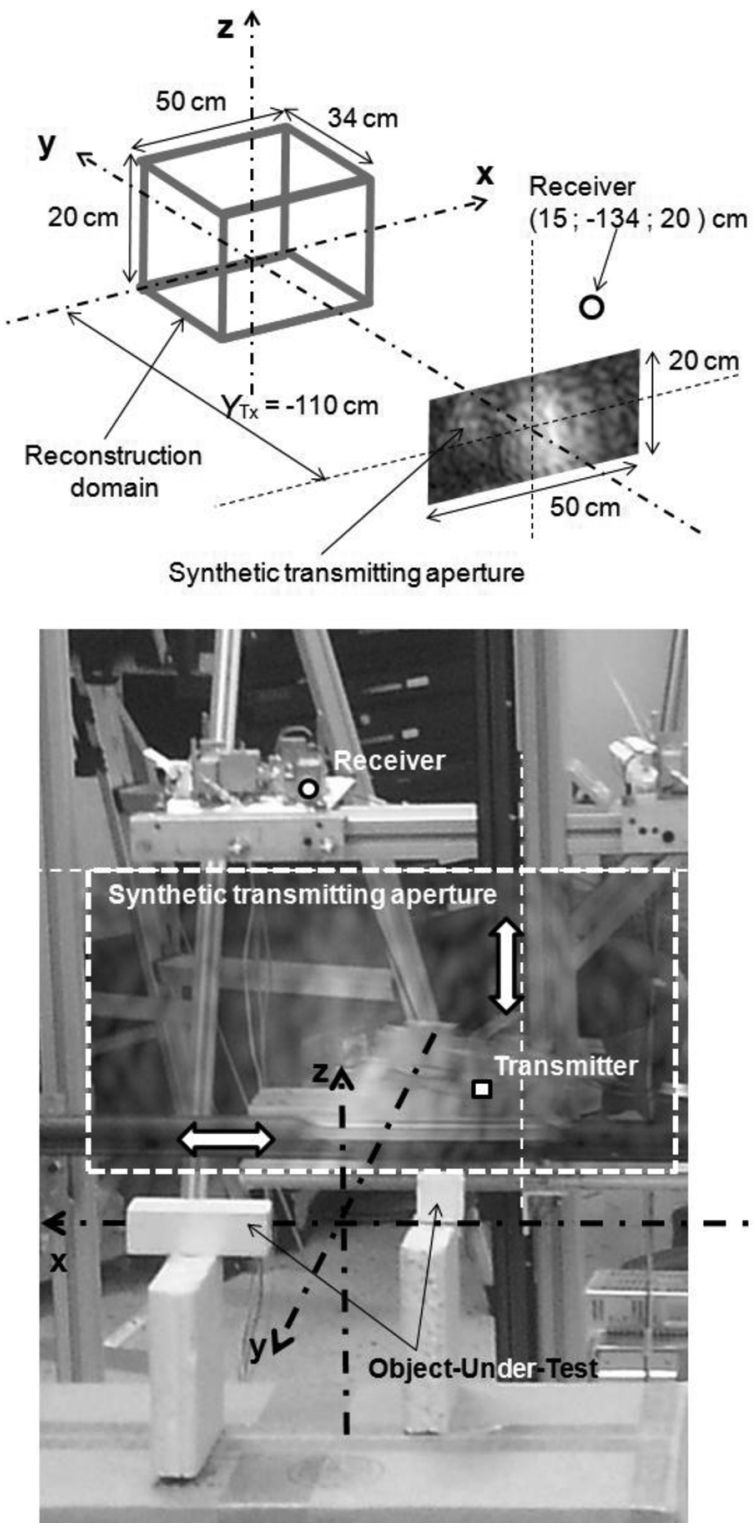

Fig. 14. Top: layout of the measurement setup. Bottom: photograph of the measurement setup with synthetic transmitting aperture and metallic OUT. Measured field amplitude (from -30 to $0 \mathrm{~dB}$ ) is shown with partial transparency. Note that this multistatic radar system has a moving transmitter and stationary receiver.

The Fourier-based method is applied again by specifying phase at the OUT center estimated from Fig. 16, $(x, y, z)=(2.5,-2.5,18) \mathrm{cm}$. Results are plotted in Fig. 17, noticing the better agreement with the SAR inversion, with the RMSE lowered to $-28 \mathrm{~dB}$. The Fourier calculation time is then $10 \mathrm{~s} \times 2$ (first guess + second case with better placement of the phase-center specification) $=20 \mathrm{~s}$, which is 70 times faster than SAR.

Finally, Fourier-based imaging with two phase center specification, placed at $(x, y, z)=(-5,-2.5,18) \mathrm{cm}$ and $(10,-2.5,18) \mathrm{cm}$, was tested, but it did not improve the results significantly with RMSE $=-28.5 \mathrm{~dB}$. This is due to the fact that the distance between the transmitter and the OUT is sufficiently large so the incident field behaves almost as a plane wave in the reconstruction domain. 

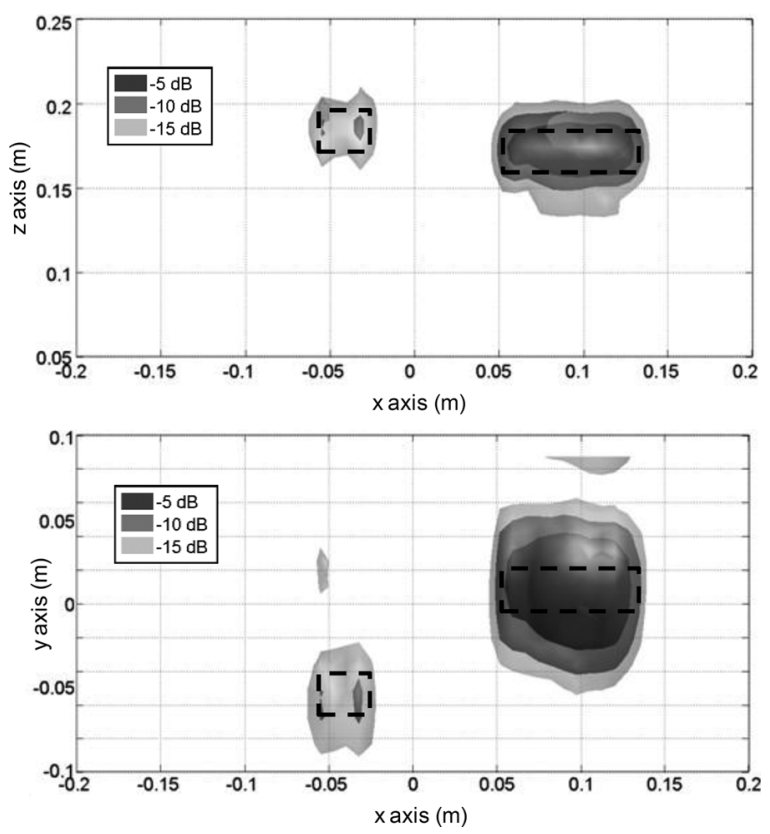

Fig. 15. Retrieved reflectivity using SAR. Amplitude isosurfaces, in decibels. Top: XZ. Bottom: XY. Black dashed lines represent the OUT profile.
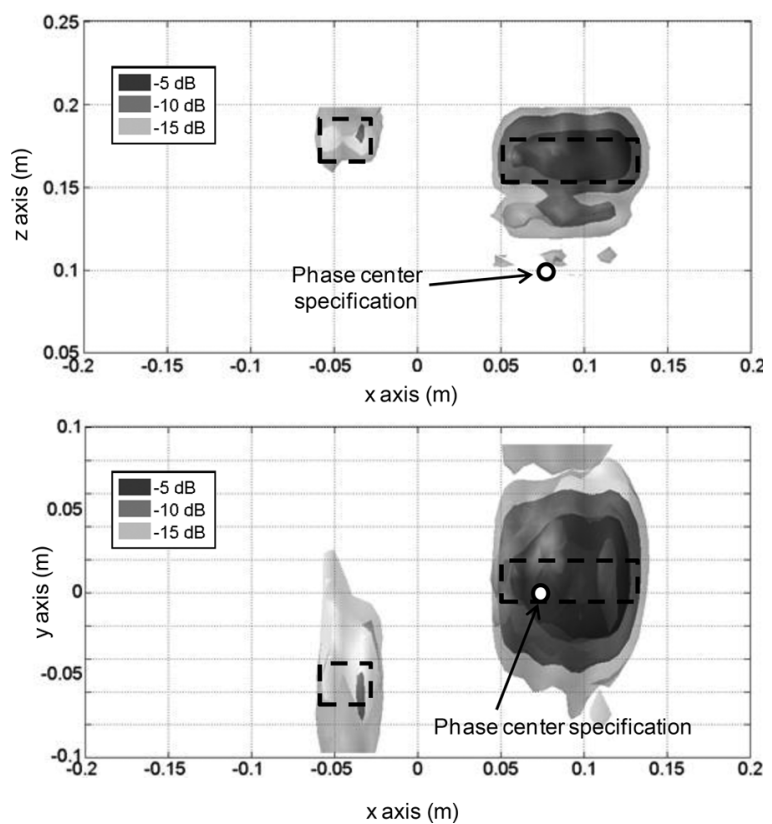

Fig. 16. Retrieved reflectivity using FFT when the phase-center specification is at $(x, y, z)=(7.5,0,10) \mathrm{cm}$. Amplitude isosurfaces, in decibels. Top: XZ. Bottom: XY. Black dashed lines represent the OUT profile.

\section{3-D SiMULATION RESULTS}

Here, we highlight the need for phase-center specification in 3-D problems. Due to mechanical limitations, this experiment cannot be carried out with the radar system presented in Section IV. Thus, 3-D simulation-based results will be used instead. Due to the large electric size of the setup, a Physical Optics code [24], [25] has been used for forward scattering problem simulation.

The problem setup is depicted in Fig. 18. The working frequency band is from 60 to $66 \mathrm{GHz}$ (the same as in [10]),
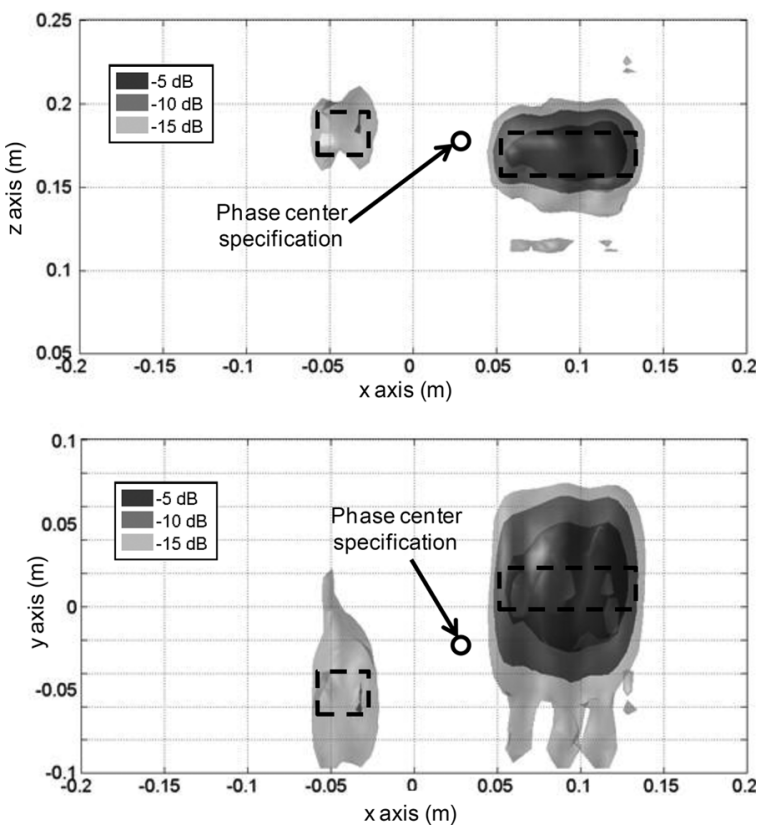

Fig. 17. Retrieved reflectivity using FFT when the phase-center specification is at $(x, y, z)=(2.5,-2.5,18) \mathrm{cm}$. Amplitude isosurfaces, in decibels. Top: XZ. Bottom: XY. Black dashed lines represent the OUT profile.

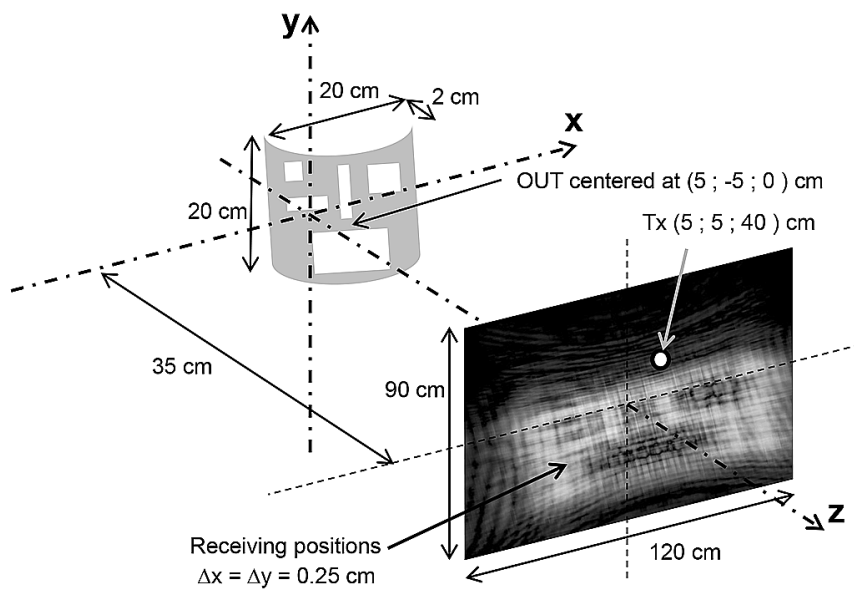

Fig. 18. Layout of the 3-D imaging system and simulated field at the receiver positions on a 2-D aperture with OUT (gray shape) positioned off center. Transmitter is represented with a white dot.

with $600-\mathrm{MHz}$ steps. The scattered field observation domain is $120 \times 90 \mathrm{~cm}$, discretized every $25 \mathrm{~mm}(0.55 \lambda$ at $66 \mathrm{GHz})$ yielding $481 \times 361$ acquisition points. The transmitter is placed $5 \mathrm{~cm}$ ahead of the observation domain for better observation of the incident field compensation distortion. Range resolution ( $z$-axis) is $25 \mathrm{~mm}$, and cross-range resolution is $15 \mathrm{~mm}$ ( $x$-axis) $\times 19 \mathrm{~mm}$ ( $y$-axis). Again, no windowing function is considered.

The OUT is a $44 \times 44 \times 4.4 \lambda$ metallic object curved about the $y$-axis, with several holes, the smallest being $1 \times 1 \mathrm{~cm}$. The OUT has been chosen so that most of the scattered power falls on the observation domain. The same computational hardware as in Section III (a laptop) was used.

The imaging domain is determined by the aperture size and sampling as well as by the $k_{\mathrm{z}}$ bandwidth and sampling. For this 


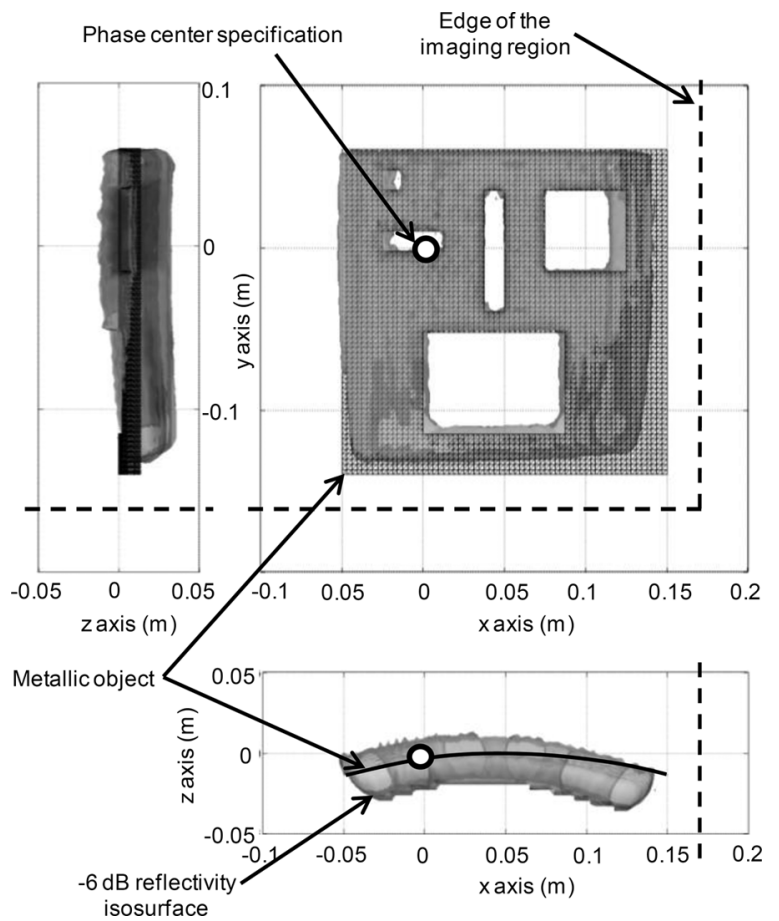

Fig. 19. Recovered reflectivity ( $-6 \mathrm{~dB}$ isosurface, in gray). $\mathrm{XY}, \mathrm{XZ}$, and $\mathrm{YZ}$ views. One phase-center specification at the origin is considered.

problem, the imaging domain is $120 \times 90 \times 25 \mathrm{~cm}$, discretized into $481 \times 361 \times 81$ points, corresponding to 14 million points. To give an idea of the size of the problem, the reflectivity on every pixel is given by the information collected across the aperture $(481 \times 361$ points $)$ for all of the 11 frequencies.

Fourier results are depicted in Figs. 19 and 20 for a case with a single and multiple phase-center specifications, respectively. In Fig. 19, the lower right corner is significantly distorted. As the incident field phase-specification error becomes larger, the reconstructed reflectivity diverges from the OUT geometry. At the lower right corner of the OUT, the distortion is $2.8 \lambda$ at the highest frequency $(66 \mathrm{GHz})$.

Distortion is compensated by introducing multiple phase center specification. Fig. 20 shows the reflectivity when 4 $\times 4$ phase center specification are considered, reducing the maximum distortion to $0.22 \lambda$. In this case, the reconstructed reflectivity more accurately represents the true OUT geometry. The positions of the phase center specification are defined from the recovered reflectivity of Fig. 19.

As a reference, SAR backpropagation results are plotted in Fig. 21. The RMSE between SAR processing and FFT with a single phase-center specification is $-17 \mathrm{~dB}$, and $-25 \mathrm{~dB}$ when 16 phase centers are considered.

Fourier-based imaging for a single phase-center specification takes $600 \mathrm{~s}, 580$ of which correspond to interpolation time ( $k$-space is discretized into $6 \times 6 \times 6$ regions). Three seconds are required for FFT and IFFT operations, with the remaining time for setting up variables and data sorting. For 16 phase-center specifications, the time is $9000 \mathrm{~s}$. A fairer comparison should take into account the time required for the first reflectivity reconstruction (Fig. 19), as the phase centers defined in Fig. 20 are based on this result. SAR backpropagation requires $252000 \mathrm{~s}$,

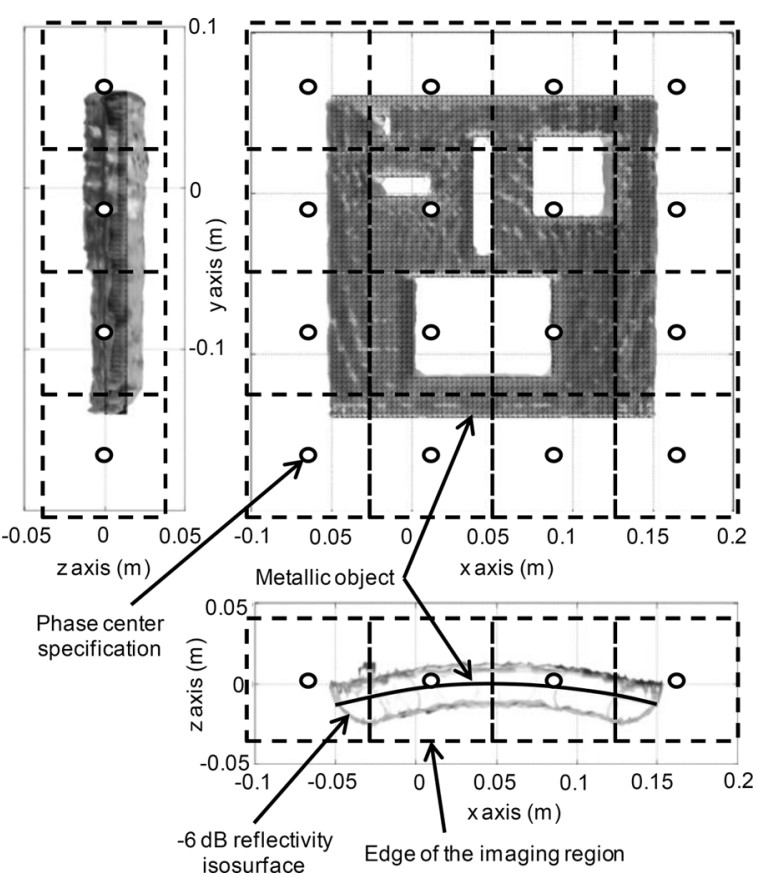

Fig. 20. Recovered reflectivity ( $-6 \mathrm{~dB}$ isosurface, in gray). $\mathrm{XY}, \mathrm{XZ}$, and $\mathrm{YZ}$ views. Sixteen phase-center specification are considered.

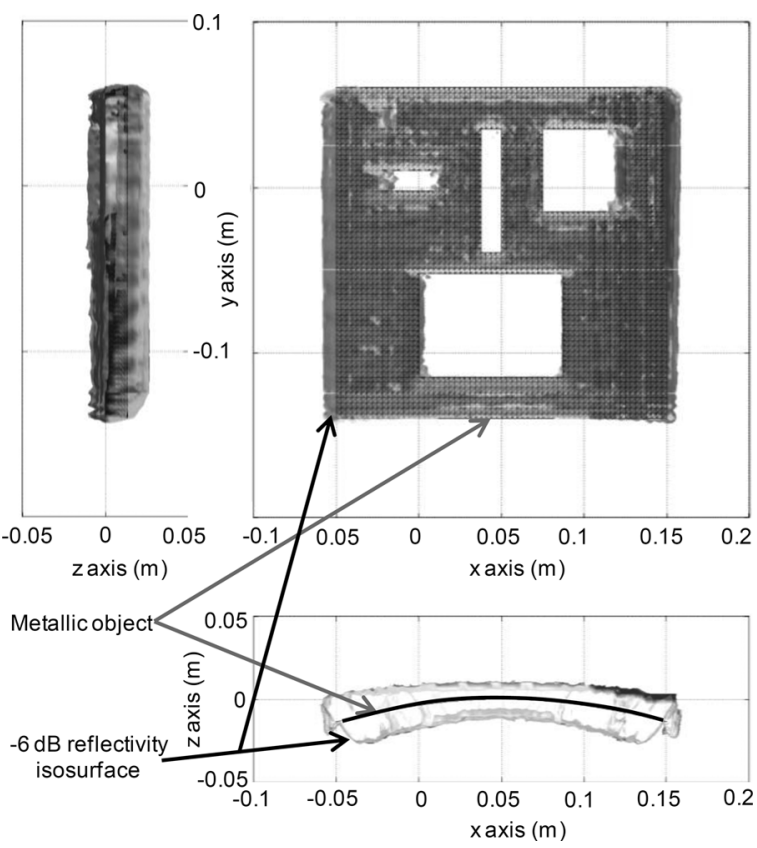

Fig. 21. SAR backpropagation results. Recovered reflectivity ( $-6 \mathrm{~dB}$ isosurface, in gray). $\mathrm{XY}, \mathrm{XZ}$, and $\mathrm{YZ}$ views.

which is 28 times slower than Fourier (26 times if the time required by the first guess reconstruction is included). Note that the smaller FFT over SAR speedup with respect to the example in Section IV is due to the need for fewer phase-center specifications in that example. Large calculation times (both for Fourier and SAR) are due to: 1) the use of single-core calculation; 3 ) the hardware itself (laptop); 3) the electrically large problem-undertest; and 5) the use of a MATLAB-based implementation. 


\section{CONCLUSION}

A Fourier-based imaging method for multistatic radar configurations has been presented. The method overcomes two of the main limitations that Fourier processing has for this radar configuration: 1) nonuniform grid in $k$-space, requiring multidimensional interpolation methods and 2) image distortion when approximating spherical wave-like OUT illumination with plane waves. The first drawback is solved by using $k$-space partitioning, applying interpolation on every domain. The second takes advantage of using multiple incident plane waves, each referred to a phase-center specification within the imaging domain.

Both solutions are fully parallelizable, thus allowing calculation time savings: multicore computers and GPU hardware are suitable for this purpose.

The Fourier-based method has been validated through 2-D-, 3-D-simulation-based, and measurement results. After benchmarking with well-known SAR backpropagation, imaging RMSE was below $-25 \mathrm{~dB}$ with 22 time speedup for the worst-case scenario.

\section{REFERENCES}

[1] E. C. Fear, X. Li, S. C. Hagness, and M. A. Stuchly, "Confocal microwave imaging for breast cancer detection: localization of tumors in three dimensions," IEEE Trans. Biomed. Eng., vol. 49, no. 8, pp. 812-822, Aug. 2002.

[2] J. A. Martínez-Lorenzo, C. M. Rappaport, and F. Quivira, "Physical limitations on detecting tunnels using underground-focusing spotlight synthetic aperture radar," IEEE Trans. Geosci. Remote Sensing, vol. 49, no. 1, pp. 65-70, Jan. 2011.

[3] D. M. Sheen, D. L. McMakin, and T. E. Hall, "Three-dimensional millimeter-wave imaging for concealed weapon detection," IEEE Trans. Microw. Theory Techn., vol. 49, no. 9, pp. 1581-1592, Sep. 2001.

[4] J. A. Martinez-Lorenzo, F. Quivira, and C. M. Rappaport, "SAR imaging of suicide bombers wearing concealed explosive threats," Progr. Electromagn. Res., vol. 125, pp. 255-272, 2012.

[5] D. L. Mensa, High Resolution Radar Cross-section Imaging. Norwood, MA, USA: Artech House, 1991.

[6] S. Lockwood, A. Brown, and H. Lee, "Backward propagation image reconstruction techniques for bistatic synthetic-aperture radar imaging systems with circular-aperture configurations," in Proc. 35th Asilomar Conf. Signals, Syst. Computers, Pacific Grove, CA, USA, 2001, vol. 1, pp. $110-115$.

[7] M. D. Desai and W. K. Jenkins, "Convolution backprojection image reconstruction, for spotlight mode synthetic aperture radar," IEEE Trans. Image Process., vol. 1, no. 4, pp. 505-517, Oct. 1992.

[8] W. G. Carrara, R. S. Goodman, and R. M. Majewski, Spotlight Synthetic Aperture Radar: Signal Processing Algorithms. Boston, MA, USA: Artech House, 1995.

[9] M. Soumekh, Synthetic Aperture Radar Signal Processing: With MATLAB Algorithms. New York, NY, USA: Wiley-Interscience, 1999.

[10] Y. Álvarez, B. Gonzalez-Valdes, J. Á. Martínez-Lorenzo, F. Las-Heras, and C. M. Rappaport, "3D whole body imaging for detecting explosive-related threats," IEEE Trans. Antennas Propag., vol. 60, no. 9, pp. 4453-4458, Sep. 2012.

[11] K. B. Cooper, R. J. Dengler, N. Llombart, B. Thomas, G. Chattopadhyay, and P. H. Siegel, "THz imaging radar for standoff personnel screening," IEEE Trans. THz Sci. Technol., vol. 1, no. 1, pp. 169-182, Sep. 2011

[12] F. Friederich, W. v. Spiegel, M. Bauer, F. Meng, M. Thomson, S. Boppel, A. Lisauskas, B. Hils, V. Krozer, A. Keil, T. Loffer, R. Henneberger, A. Huhn, G. Spickermann, P. Bolivar, and H. Roskos, "THz active imaging systems with real-time capabilities," IEEE Trans. THz Sci. Technol., vol. 1, no. 1, pp. 183-200, Sep. 2011.
[13] D. M. Sheen, D. L. McMakin, H. D. Collins, T. E. Hall, and R. H Severtsen, "Concealed explosive detection on personnel using a wideband holographic millimeter-wave imaging system," in Proc. SPIE, Signal Process., Sensor Fusion, and Target Recognition V., Orlando, FL, USA, 1996, vol. 2755, pp. 503-513.

[14] J. L. Fernandes, R. Obermeier, M. Hagelen, J. A. Martínez-Lorenzo, and C. M. Rappaport, "A comparison of experimental and modeled results of an active millimeter wave inverse synthetic aperture radar system used to perform standoff detection of person-borne improvised explosive devices," in Proc. IEEE Int. Conf. Technol. Homeland Security, Waltham, MA, USA, 2010, pp. 428-434.

[15] G. Yates, A. M. Horne, A. Blake, R. Middleton, and D. Andre, "Bistatic SAR image formation," IEEE Proc. on Radar Sonar Navigation, vol. 153, no. 3, pp. 208-213, 2006.

[16] M. Soumekh, "Bistatic synthetic aperture radar inversion with application in dynamic object imaging," IEEE Trans. Signal Process., vol. 39, no. 9, pp. 2044-2055, Sep. 1991.

[17] I. Stojanovic, W. C. Karl, and M. Cetin, "Compressed sensing of monostatic and multi-static SAR," in Proc. SPIE, Algorithms for Synthetic Aperture Radar Imagery XVI, Orlando, FL, USA, 2009, vol. 7337, pp. $5-12$.

[18] Y. F. Shao, R. Wang, Y. K. Deng, Y. Liu, R. Chen, G. Liu, and O. Loffeld, "Fast backprojection algorithm for bistatic SAR imaging," IEEE Geosci. Remote Sensing Lett., vol. 10, no. 5, pp. 1080-1084, Sep. 2013.

[19] G. Halcrow and B. Mulgrew, "Nonnlinear $k$-space mapping method for SAR Fourier imaging," in Proc. IEEE RADAR Conf., Verona, NY, USA, 2006, pp. 384-387.

[20] J. Shaeffer, B. A. Cooper, K. W. Hom, R. C. Baucke, and N. A. Talcott Jr., "A review of bistatic $k$-Space imaging for electromagnetic prediction codes for scattering and antennas," IEEE Antennas Propag. Mag., vol. 39, no. 5, pp. 21-29, Oct. 1997.

[21] H. Buddendick and T. F. Eibert, "Bistatic image formation from shooting and bouncing rays simulated current distributions," Progr. Electromagn. Res., vol. 119, pp. 1-18, 2011.

[22] Y. Álvarez, J. Laviada, L. Tirado, C. García, J. A. Martínez, F. LasHeras, and C. M. Rappaport, "Inverse fast multipole method for monostatic imaging applications," IEEE Geosci. Remote Sensing Lett., vol. 10, no. 5, pp. 1239-1243, Sep. 2013.

[23] C. A. Balanis, Advanced Engineering Electromagnetics. New York, NY, USA: Wiley, 1989.

[24] J. G. Meana, J. A. Martínez-Lorenzo, and F. Las-Heras, "High frequency techniques: the physical optics approximation and the modified equivalent current approximation (MECA)," in Electromagnetic Waves Propagation in Complex Matter, P. A. Kishk, Ed. : InTech.

[25] J. G. Meana, J. A. Martinez-Lorenzo, F. Las-Heras, and C. M. Rappaport, "Wave scattering by dielectric and lossy materials using the modified equivalent current approximation (MECA)," IEEE Trans. Antennas Propag., vol. 58, no. 11, pp. 3757-3761, Nov. 2010.

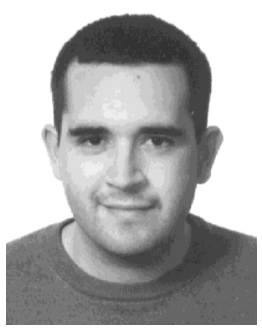

Yuri Alvarez (S'06-M'09) was born in Langreo, Spain, in 1983. He received the M.S. and Ph.D. degrees in telecommunication engineering from the University of Oviedo, Asturias, Spain, in 2006 and 2009, respectively.

He was a Visiting Scholar with the Department of Electrical Engineering and Computer Science, Syracuse University, Syracuse, NY, USA, in 2006 and 2008; and a Visiting Postdocoral with the Gordon Center for Subsurface Sensing and Imaging Systems (CenSSIS) - ALERT Center of Excellence, Northeastern University, Boston, MA USA, in 2011, 2012, and 2013. He is currently an Assistant Professor with the Signal Theory and Communications, University of Oviedo, Gijón, Spain. His interests and research studies have been focused on antenna diagnostics, antenna measurement techniques, RF techniques for indoor location, and inverse scattering and imaging techniques.

Prof. Alvarez was the recipient of the 2011 Regional and National Awards to the Best Ph.D. Thesis on Telecommunication Engineering. 


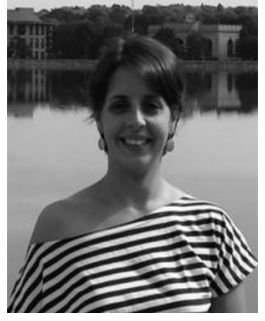

Yolanda Rodriguez-Vaqueiro (S'13) received the B.S. and M.S. degrees in electrical engineering from the University of Vigo, Vigo, Spain. She is currently working toward the Ph.D. degree in electrical engineering at Northeastern University, Boston, MA, USA.

She is a Research Assistant with ALERT (Awareness and Localization of Explosive Related Threats), Northeaster University, Boston, MA, USA. She has authored 14 journal and conference papers. Her areas of interest include the application of compressive sensing techniques to inverse electromagnetic problems, signal processing in medical applications, and electromagnetics.

Mrs. Rodriguez-Vaqueiro was the recipient of the Best Paper Award at the 2012 IEEE Homeland Security Conference and the 2014 EuCAP Conference, and she was awarded an Honorable Mention in the Student Paper Competition at the 2013 IEEE APS/URSI Conference.

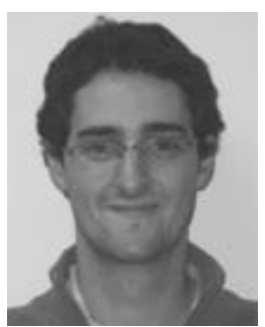

Borja Gonzalez-Valdes (M'09) received the Electrical Engineering degree and Ph.D. degree from the University of Vigo, Vigo, Spain, in 2006 and 2010, respectively.

From 2006 to 2010, he was with the Antenna and Optical Communications Group, University of Vigo. During 2008 and 2009, he was a Visiting Researcher with the Gordon CenSSIS, Northeastern University, Boston, MA. USA. In 2011, he joined the ALERT Center of Excellence, Northeastern University. His research interests include antenna design, inverse scattering, radar, advanced imaging techniques, and $\mathrm{THz}$ technology.

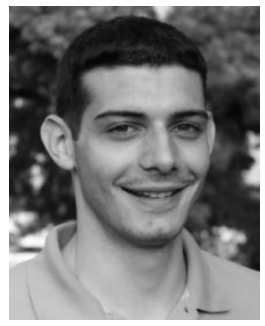

Spiros Mantzavinos received the B.S. and M.S degrees in electrical and computer engineering from Northeastern University, Boston, MA, USA.

$\mathrm{He}$ is currently an Engineer with Analogic Corporation, where he works with a small team of engineers on a medical imaging device. Starting in 2009, he worked with the ALERT (Awareness and Localization of Explosives Related Threats) team, Northeastern University, Boston, MA, USA, developing a next-generation millimeter-wave, whole-body imaging system. Alongside his graduate research, Spiros graduated from the Gordon Engineering Leadership Program at Northeastern University in 2012.

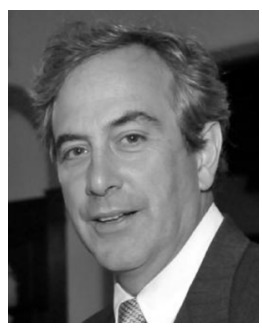

Carey M. Rappaport (SM'96-F'06) received the $\mathrm{SB}$ degree in mathematics and the SB, SM, EE, and $\mathrm{Ph} . \mathrm{D}$. degrees in electrical engineering from the Massachusetts Institute of Technology, Cambridge, MA, USA, in 1982 and 1987, respectively.

He was a Teaching and Research Assistant with Massachusetts Institute of Technology, Cambridge, MA, USA, from 1981 until 1987, and during the summers at COMSAT Labs, Clarksburg, MD, USA, and The Aerospace Corporation, El Segundo, CA, USA. He joined the faculty at Northeastern University, Boston, MA, USA, in 1987. He has been a Professor of electrical and computer engineering since July 2000. In 2011, he was appointed College of Engineering Distinguished Professor. During the fall of 1995, he was a Visiting Professor of electrical engineering with the Electromagnetics Institute, Technical University of Denmark, Lyngby, Denmark, as part of the W. Fulbright International Scholar Program. During the second half of 2005, he was a Visiting Research Scientist with the Commonwealth Scientific Industrial and Research Organisation (CSIRO), Epping, Australia. He has consulted for CACI, Alion Science and Technology, Inc., Geo-Centers, Inc., PPG, Inc., and several municipalities on wave propagation and modeling, and microwave heating and safety. He was Principal Investigator of an ARO-sponsored Multidisciplinary University Research Initiative on Humanitarian Demining, Co-Principal Investigator of the National Science Foundation-sponsored Engineering Research Center for Subsurface Sensing and Imaging Systems (CenSSIS), and Co-Principal Investigator and Deputy Director of the DHS-sponsored Awareness and Localization of Explosive Related Threats (ALERT) Center of Excellence. He has authored or coauthored over 400 technical journal and conference papers in the areas of microwave antenna design, electromagnetic wave propagation and scattering computation, and bioelectromagnetics, and has received two reflector antenna patents, two biomedical device patents and four subsurface sensing device patents.

Prof. Rappaport is a member of Sigma Xi and Eta Kappa Nu. He was the recipient of the IEEE Antenna and Propagation Society's H.A. Wheeler Award for Best Applications Paper as a student in 1986.

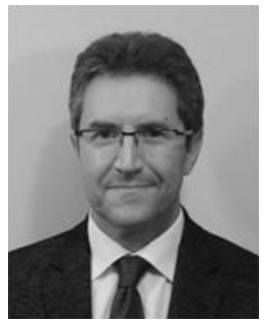

Fernando Las-Heras (M'86-SM'08) received the M.S. and Ph.D. degrees in telecommunication engineering from the Technical University of Madrid, Madrid, Spain, in 1987 and 1990, respectively.

From 1988 to 1990, he was a National Graduate Research Fellow. From 1991 to 2000, he was an Associate Professor with the Department of Signal, Systems and Radiocommunications, Technical University of Madrid, Madrid, Spain. Since December 2003, he has been a Full Professor with at the University of Oviedo, where he has been Vice-dean for Telecommunication Engineering at the Technical School of Engineering at Gijón from 2004 to 2008. From 2001 he heads the research group Signal Theory and Communications TSC-UNIOVI at the Dept. of Electrical Engineering of the University of Oviedo, Gijón, Spain, as the Principal Investigator in several projects of national programs and contracts with companies. He was a Visiting Lecturer with the National University of Engineering in Peru in 1996, a Visiting Researcher with Syracuse University in 2000, and short-term Visiting Lecturer with ESIGELEC in France since 2005. Since 2005, he has held the Telefónica Chair ICTs applied to Environment and Climate Change at the University of Oviedo, and, since 2010, he has been a member of the Science, Technology and Innovation Council of Asturias, Spain. He has authored and coauthored over 300 technical journal and conference papers in the areas of antenna design, the inverse electromagnetic problem with application to diagnostic, measurement and synthesis of antennas, propagation and computational electromagnetics, and engineering education.

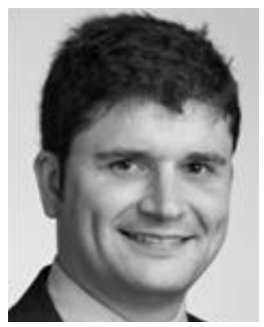

Jose A. Martinez-Lorenzo (S'03-M'05) received the joint B.S/M.S. and Ph.D. degrees from the University of Vigo, Vigo, Spain, in 2002 and 2005, respectively, both in electrical engineering.

He joined the faculty of the University of Oviedo, Gijon, Spain, in 2004, where he was an Assistant Professor with the Department of Signal Theory and Communications. In 2006, he joined the Bernard $M$ Gordon Center for Subsurface Sensing and Imaging Systems, Northeastern University, Boston, MA, USA. In 2010, he was appointed a Research Assistant Professor with the Electrical and Computer Engineering (ECE) Department, Northeastern University. Since August 2013, he has held a joint appointment with the Departments of Mechanical and Industrial Engineering and ECE as a Tenure-Track Assistant Professor. He is an active member of ALERT (Awareness and Localization of Explosives-Related Threats), a DHS Center of Excellence awarded to Northeastern. He has authored and coauthored over 140 technical journal and conference papers. His research is geared towards the understanding, modeling and solving complex engineering problems, with an emphasis on mechanical and electromagnetic sensing and imaging methods for security and biomedical applications (i.e., explosive detection and breast cancer detection).

Prof. Martinez-Lorenzo led the team that won the Best Paper Award at the 2012 IEEE Conference on Technologies for Homeland Security for the paper on "A compressed sensing approach for detection of explosive threats at standoff distances using a Passive Array of Scatterers." 